\title{
FTY720 reactivates cryptococcal granulomas in mice through S1P receptor 3 on macrophages
}

\author{
Arielle M. Bryan, ${ }^{1}$ Jeehyun Karen You, ${ }^{1}$ Travis McQuiston, ${ }^{2}$ Cristina Lazzarini, ${ }^{1}$ Zhijuan Qiu, ${ }^{1}$ Brian Sheridan, ${ }^{1}$ \\ Barbara Nuesslein-Hildesheim, ${ }^{3}$ and Maurizio Del Poeta ${ }^{1,4,5}$
}

'Department of Microbiology and Immunology, Stony Brook University, Stony Brook, New York, USA. ${ }^{2}$ FirstString Research Inc., Mount Pleasant, South Carolina, USA. ${ }^{3}$ Novartis Institute for Biomedical Research, Novartis Pharma AG, Basel, Switzerland. “Division of Infectious Diseases, School of Medicine, Stony Brook University, Stony Brook, New York, USA. ${ }^{5}$ Veterans Affairs Medical Center, Northport, New York, USA.

\begin{abstract}
FTY720 is a treatment for relapsing remitting multiple sclerosis (MS). It is an analog of sphingosine-1-phosphate (S1P) and targets S1P receptors 1, 3, 4, and 5. Recent reports indicate an association between long-term exposure to FTY720 and cases of cryptococcal infection. Here, we studied the effect of FTY720 and its derivative, BAF312, which only target S1P receptors 1 and 5, in a mouse model of cryptococcal infection. We found that treatment with FTY720, but not with BAF312, led to decreased survival and increased organ burden in mouse cryptococcal granulomas. Both FTY720 and BAF312 caused a profound $\mathrm{CD}^{+}$and $\mathrm{CD8} 8^{+} \mathrm{T}$ cell depletion in blood and lungs but only treatment with FTY720 led to cryptococcal reactivation. Treatment with FTY720, but not with BAF312, was associated with disorganization of macrophages and with M2 polarization at the granuloma site. In a cell system, FTY720 decreased phagocytosis and production of reactive oxygen species by macrophages, a phenotype recapitulated in the S1pr3-/- knockout macrophages. Our results suggest that FTY720 reactivates cryptococcosis from the granuloma through a S1P receptor 3-mediated mechanism and support the rationale for development of more-specific receptor modulators for therapeutic use of MS.
\end{abstract}

\section{Introduction}

Cryptococcus spp. are important human fungal pathogens that cause life-threatening meningo-encephalitis in severely lymphopenic patients $(1,2)$. Cryptococcus neoformans causes approximately 223,100 cases of meningitis every year, resulting in 181,000 deaths worldwide (3). Cryptococcal meningitis occurs mainly in patients with HIV infections, but in the United States there is an increasing number of non-HIV cases associated with comorbidities such as hydrocephalus and renal failure. Individuals are also predisposed to non-HIV cryptococcal meningitis by transplantation, combined T and B cell defects, Cushing's syndrome, liver disease, and hypogammaglobulinemia (4). There is evidence suggesting that subclinical pulmonary infection occurs in nonimmunocompromised hosts to contain cryptococcal cells within granulomas, which may later reactivate upon immunosuppression to cause a clinical disease (5-8). Despite the substantial mortality and clinical challenge of treating cryptococcal meningo-encephalitis, there is still much that is unknown about the delicate interactions between the host immune response and fungal pathogens like C. neoformans.

In recent years, cases of cryptococcal meningitis have been reported for FTY720 (Gilenya, Novartis) in the postmarketing

Authorship note: $\mathrm{AMB}$ and JKY are co-first authors and contributed equally. Conflict of interest: MDP is a cofounder and Chief Scientific Officer of MicroRid Technologies Inc. TMQ is the Director of Translational Research at FirstString Inc. $\mathrm{BNH}$ is a director at Novartis Institutes for BioMedical Research. Copyright: ( 2020 , American Society for Clinical Investigation. Submitted: December 30, 2019; Accepted: May 15, 2020; Published: July 27, 2020 Reference information: J Clin Invest. 2020;130(9):4546-4560. https://doi.org/10.1172/JCl136068. setting after approximately 2 to 3 years of treatment, although an exact relationship with the duration of treatment is unknown. The cumulative reporting rate, including all cases of cryptococcal meningitis received through February 28, 2019, is $7 / 100,000$ patient years (95\% CI: 5/100,000-10/100,000 patient years) (our unpublished observations). Treatment options include induction therapy of intravenous amphotericin B and oral flucytosine followed by a long-term (at least 10 weeks) maintenance therapy of oral fluconazole. Due to these incidences, cryptococcal meningitis has been added to the safety warning and precautions in the prescribing information for FTY720 (9).

FTY720 is a pro-drug that is phosphorylated in vivo where the phosphorylated form is an analog of sphingosine-1-phosphate (S1P), an important host-signaling lipid (10-12). FTY720-phosphate (FTY72OP) targets S1P receptors 1, 3, 4, and 5 (S1PR1, S1PR3, S1PR4, and S1PR5) (13-16). The compound works to treat multiple sclerosis (MS) through S1PR1, which is internalized upon FTY720P binding, making S1PR1 unresponsive to the natural ligand S1P, working as a functional antagonist on $\operatorname{S1PR1}(17,18)$. By targeting S1PR1, FTY720P prevents lymphocytes from exiting lymph nodes, resulting in a substantial decrease of peripheral lymphocytes and preventing autoaggressive lymphocytes from crossing the blood-brain barrier. This prevents their pathological effect on inflamed central nervous system (CNS) tissues during MS $(18,19)$. BAF312 (Mayzent, Novartis) was developed as a morespecific analog of FTY720, as it targets only S1PR1 and S1PR5 (20-22). Unlike FTY720, BAF312 does not need to be phosphorylated due to the addition of an amino carboxylic acid moiety, which mimics the amino phosphate in FTY720P (21). Additionally, the hydrophobic alkyl chain of FTY720 was altered to increase the specificity of the molecule such that S1PR1 agonism occurs to 
mediate the same therapeutic outcome of lymphocyte redistribution without producing S1PR3-mediated effects $(20,21)$.

Previous work in our lab revealed the importance of the S1P signaling pathway in the granulomatous response to an attenuated $C$. neoformans strain ( $\triangle g c s 1)$, a mouse model that forms lung granulomas strikingly similar to the granulomas found in humans (23-25). Formation of granulomas in response to C. neoformans $\Delta g c s 1$ infection was dependent on sphingosine kinase 1 (SK1) and associated with elevated levels of S1P, MCP1, and TNF- $\alpha$ in the bronchoalveolar lavage fluid of C. neoformans $\Delta g c s 1$-infected mice (24-26).

Using this mouse model, here we show that FTY720, but not BAF312, reactivates $C$. neoformans infection from the granuloma and that this effect may be mediated by the specific action of FTY720P on S1PR3 on macrophage functions. This work also suggests that reduced lymphocyte counts alone may not be sufficient for the increased susceptibility to C. neoformans and that cryptococcal reactivation most likely occurs when macrophages at the site of the granuloma are no longer able to contain and neutralize fungal cells.

\section{Results}

FTY72O affects survival and organ burden in a $C$. neoformans reactivation model. To study the effect of FTY720 on cryptococcal infection in the mouse model, the attenuated strain C. neoformans $\Delta g c s 1$ was used. When this strain is inhaled by either WT C57BL6/J or $\mathrm{CBA} / \mathrm{J}$ mouse, within 30 days animals develop lung granulomas similar to humans (23-25) and survive cryptococcal infection. Thirty days after the mice were infected, they either received the vehicle control $\left(\mathrm{H}_{2} \mathrm{O}\right)$ or $1 \mathrm{mg} / \mathrm{kg} /$ day of FTY720 or BAF312 via gavage. Mice survival was subsequently monitored. We found that treatment with FTY720 led to $50 \%$ of mice succumbing to C. neoformans $\triangle g c s 1$ (Figure 1A). There was no change in survival when FTY720 was administered to uninfected mice (data not shown). Mice that survived the treatment with FTY720 were sacrificed at day 50 after compound administration and fungal burden was examined. We found a significant increase in the lung burden in FTY720-treated mice and approximately $50 \%$ of the mice brains examined in the FTY720-treated group had fungal burden in the brain (Figure 1, B and C). In addition, surviving mice from the FTY720-treated group had signs and symptoms of disseminated cryptococcal disease: weight loss (although not greater than $20 \%$ ), less physical activity, and mild tremor. As expected, vehicle-treated mice (control) sacrificed at day 50 after compound administration and examined for fungal burden had an average number of colony forming units (CFUs) that was similar to the number of cells the mice were originally infected with intranasally ( $\left.\log _{10} 5.7\right)$, and only 2 of 11 mice showed some fungal cells in the brain tissue (Figure 1, B and C). Surprisingly, mice treated with BAF312 showed a survival comparable to control mice that were vehicle-treated (Figure 1A). Their organ burden was also similar to that of the control mice (Figure 1, B and C). Control or BAF312-treated mice showed normal physical activity, no weight loss, or clinical signs of cryptococcosis. These results suggest that, in contrast to FTY720, BAF312 did not induce cryptococcal cell replication in the lung. As such, mice were still able to contain the fungus within the granuloma.
Histological analysis via $\mathrm{H} \& \mathrm{E}$ and mucicarmine stains of lungs isolated from mice treated with FTY720 showed a less well-contained granuloma structure (Figure 1D, top 2 rows) with C. neoformans cells stained magenta (Figure 1D, bottom row). C. neoformans cells were also found outside of the granuloma structure in FTY720-treated mice (Figure 1D, inset). In contrast, the granuloma structure of the mice treated with BAF312 was similar to the control mice with minimal, if any, fungal cells found outside the granuloma (Figure 1D). In general, these results corroborate the lung organ burden data and they also suggest that host immune response in mice treated with FTY720 is now less capable of controlling fungal replication within the granuloma, which eventually leads to the escape of fungal cells.

To gather insights into the effect of the compounds on the primary infection model, we administered the compounds beginning 2 days before infection with $C$. neoformans. For this experiment, we used the low virulent $C$. neoformans $\triangle g c s 1$ and the more virulent clinical isolate $C$. neoformans WT H99. When mice were infected with $C$. neoformans $\Delta g c s 1$, we did not see any difference in mice survival (Supplemental Figure 1A; supplemental material available online with this article; https://doi.org/10.1172/JCI136068DS1) and they did not show any significant change in organ burden (Supplemental Figure 1, B and C). However, lung histology of mice treated with FTY720 but not with BAF312 showed different granuloma structures (Supplemental Figure 1D). The giant macrophages containing C. neoformans were not organized in a ring surrounding the necrotic tissue but were rather dispersed within the granulomatous structure, suggesting an uncoordinated lung host response in the mice treated with FTY720.

When mice were infected with $C$. neoformans WT H99, we observed a significant improvement in survival when they were treated with BAF312 (Figure 2A), whereas FTY720-treated mice succumbed to the primary infection caused by WT H99 strain, similarly to the vehicle-treated (control) mice. Lung and brain fungal burden of surviving mice treated with BAF312 showed that fungal cells were still present in the lung (Figure 2B) and in the brain, although 2 mice treated with BAF312 did not have any fungal cells in the brain tissue (Figure $2 \mathrm{~B}$ ). We did not perform histology on mice infected with C. neoformans WT H99 because granulomas do not develop in this model.

We then investigated whether the 2 drugs have any antifungal activity. We found that FTY720 and BAF312 possess antifungal activity against $C$. neoformans and C. gattii, and FTY720 also exerts activity against Candida albicans (Table 1). Furthermore, the phosphorylated form of FTY720 (FTY720P) completely loses antifungal activity (Table 1). Interestingly, C. neoformans cells cannot phosphorylate FTY720 as mammalian cells do (Figure 2C). These results suggest that BAF312 exerts antifungal activity in vitro and in our animal infection model.

FTY72O and BAF312 both affect circulating T cells. Both FTY720 and BAF312 are known to cause profound lymphopenia. To examine how FTY720 and BAF312 affect immune cell recruitment to the lung, flow cytometric analyses of blood and lung single-cell suspensions were performed during the course of the reactivation model of infection. T cells, monocytes, and neutrophils were quantified in the blood whereas T cells, alveolar and recruited macrophages, monocytes, dendritic cells, and neutrophils were quantified in the 
A

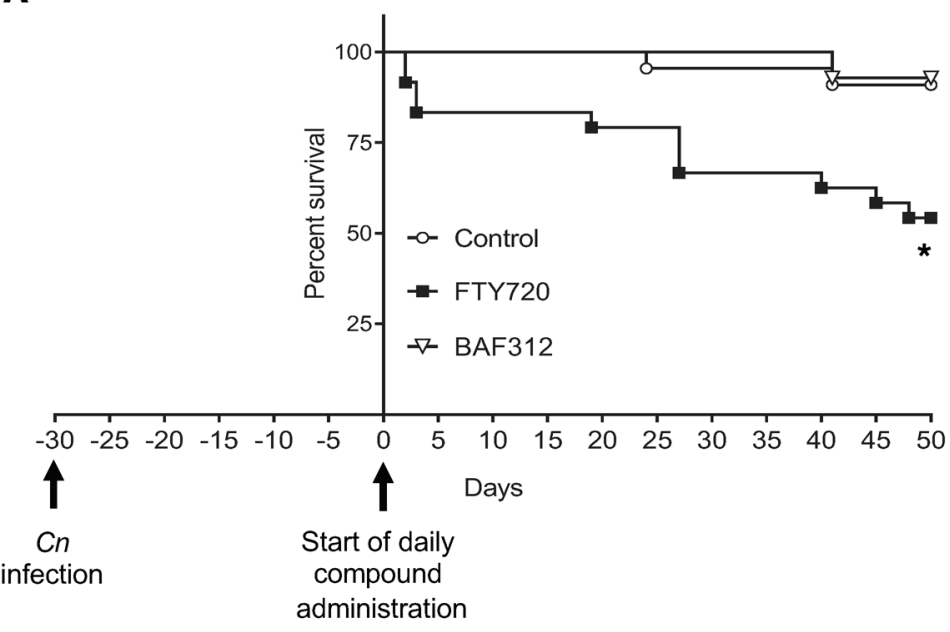

C

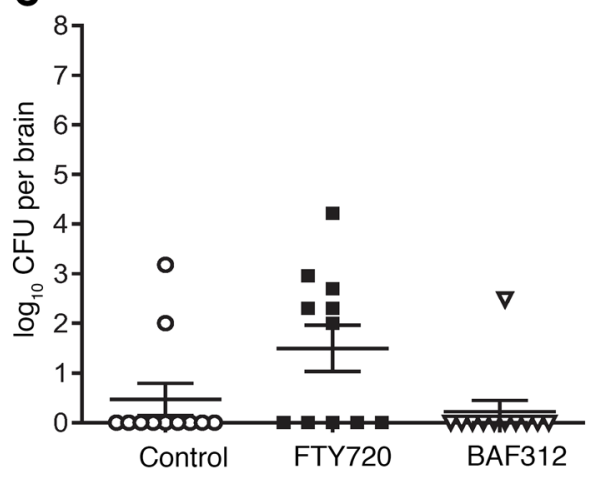

D

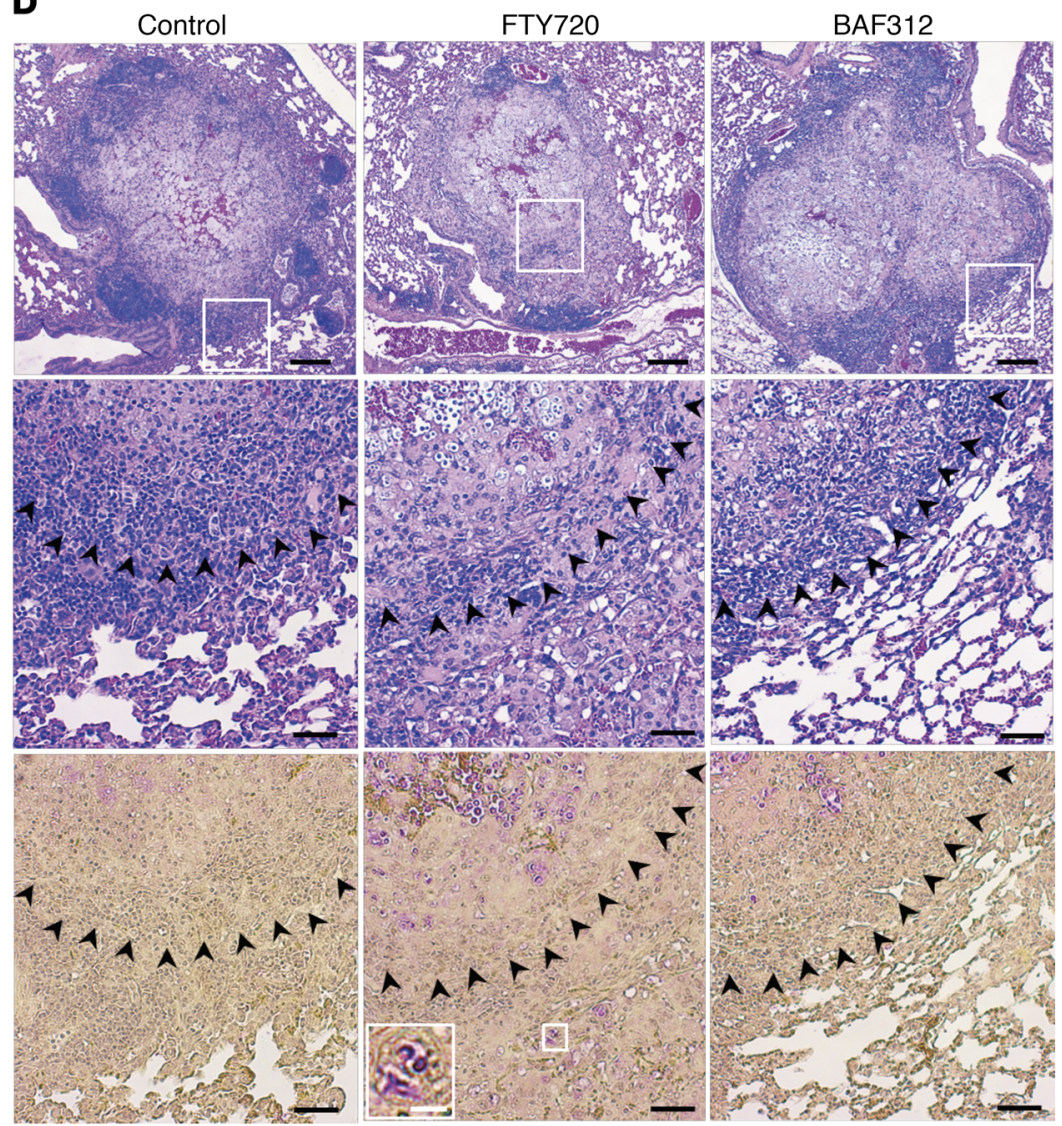

B

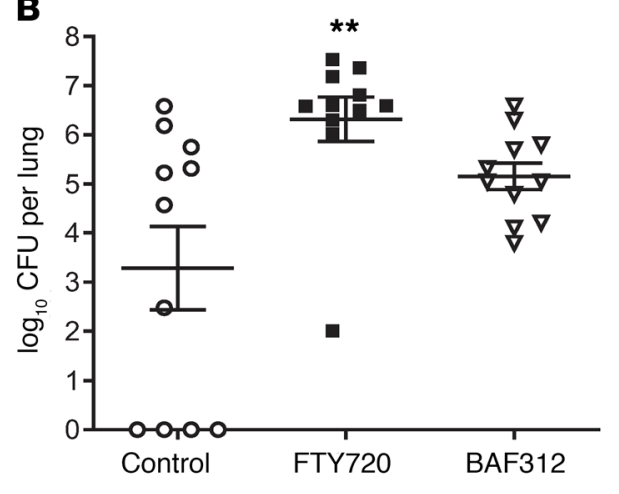

Figure 1. Mice treated with FTY720 30 days after infection have decreased survival and an increase in C. neoformans growth in granulomas. (A) Mice were infected for 30 days with C. neoformans $\Delta$ gcs 1 before daily compound oral administration and survival of mice was monitored. All compounds were given at a dose of $1 \mathrm{mg} / \mathrm{kg} /$ day. Survival curves were compared using the log-rank (Mantel-Cox) test. For FTY720, $n=16$ mice; BAF312, $n=14$ mice; and vehicle control $\left(\mathrm{H}_{2} \mathrm{O}\right), n=14$ mice. ${ }^{*} P=$ 0.0025 . (B and $\mathbf{C}$ ) After 50 days of daily compound administration or when mice lost more than $20 \%$ body weight, mice were sacrificed and organs were analyzed for CFUs. For FTY720, $n=11$ mice; BAF312, $n=11$ mice; and vehicle control $\left(\mathrm{H}_{2} \mathrm{O}\right), n=11$ mice. Organ burden was compared using 1-way ANOVA with Bonferroni's multiple comparisons post hoc test. $P$ values were corrected for multiplicity using the Bonferroni's adjustment. ${ }^{* *} P=0.0016$. All error bars represent SEM. (D) After 50 days of daily compound administration, 4 lungs were isolated for histology using H\&E stain (top 2 rows) and mucicarmine (bottom row). C. neoformans cells stain magenta in mucicarmine. Scale bars: $200 \mu \mathrm{m}$ (top row), $50 \mu \mathrm{m}$ (middle and bottom rows), and $12.5 \mu \mathrm{m}$ (inset, white bar). The white boxes indicate the enlarged area and the black arrowheads denote the border of the granuloma. 
A

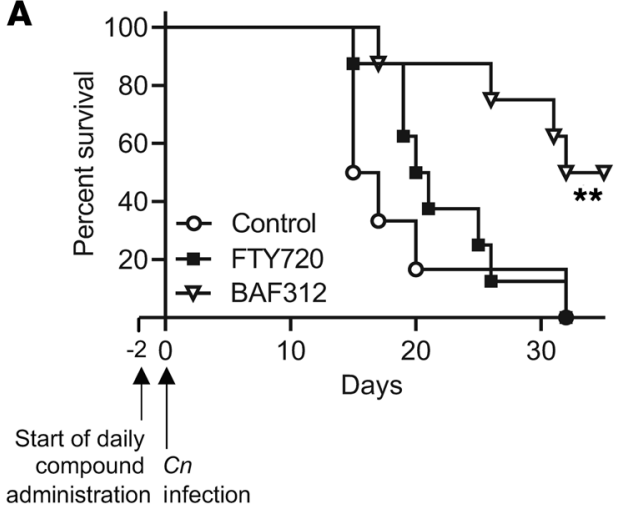

B

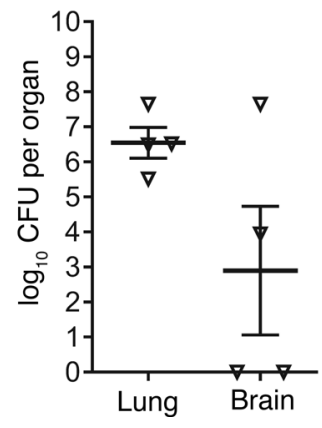

c

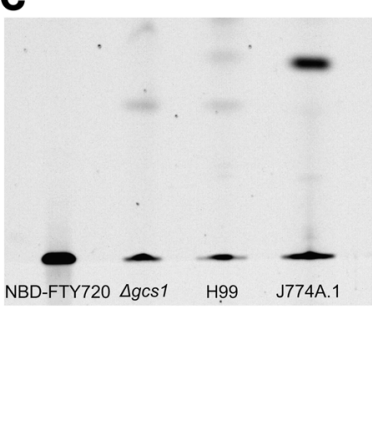

lung parenchyma of mice receiving vehicle, FTY720, or BAF312 (Supplemental Figure 2). Following the same study design as in Figure 1, samples were taken at day -1 (before compound administration) and days 5 and 20 after compound administration. As expected, both drugs caused a decrease in circulating T cells (Figure 3), suggesting that reactivation is not caused by T cell redistribution since it occurs with both compounds. Lung $\mathrm{CD} 4^{+}$and $\mathrm{CD} 8^{+}$ $\mathrm{T}$ cells were found to be reduced in the lung tissue of FTY720- and BAF312-treated mice in comparison with the vehicle-treated control mice (Figure 3). Additionally, dendritic cells (DCs) as well as lung-recruited and alveolar macrophages were decreased in response to both FTY720 and BAF312 (Figure 3). Other immune cell populations did not show significant changes in recruitment (Figure 3). These results suggest that there are no major shifts in the number of immune cells that make up the structure of the granuloma, suggesting that cryptococcal reactivation caused by FTY720 could be due to specific function(s) that the drug exerts on the intracellular pathways through the S1P receptors, leading to a disorganization of the host cells in the granuloma.
Figure 2. BAF212 increases survival in mice after primary infection with WT C.neoformans H99. (A) Mice received compound daily via gavage (FTY72O, $n=8$; BAF312, $n=8$; or vehicle control $\left[\mathrm{H}_{2} \mathrm{O}\right], n=8$ ) starting 2 days before intranasal infection with $C$. neoformans $\mathrm{Hg}$ and survival of mice was monitored. Survival curves were compared using the log-rank (MantelCox). ${ }^{* *} P=0.0083$. (B) After 40 days of daily compound administration, BAF312 mice that survived were euthanized and analyzed for CFUs in the lung and the brain, $n=4$. All error bars represent SEM. (C) NBD-FTY720 was added to the media of either mammalian cells (J774A.1) or C. neoformans cells ( $\Delta$ gcs 1 and $\mathrm{H99}$ ) at a concentration of $2 \mu \mathrm{g} / \mathrm{mL}$. NBD-FTY720 was extracted from the media and evaluated via thin layer chromatography, $n=3$ independent experiments.

FTY720 alters lung cytokine profiles. To gather additional information on the effect of FTY720 and BAF312 treatment on the host immunity during the maintenance of the granuloma, we performed a thorough cytokine analysis in lung tissue. Mice were infected with C. neoformans $\Delta g c s 1$ and allowed to form lung granuloma for 30 days. Following the same study design as in Figure 3 , samples were taken at day -1 (before compound administration) and days 5 and 20 after compound administration. We did not find major differences in cytokine level that are affected by FTY720 and not by BAF312 treatment (Figure 4 and Supplemental Figure 3). However, at day 20 after compound administration, IL-2, IL-4, IL-6, IL-9, IL-10, and IL-15 were slightly decreased in FTY720-treated compared with BAF312- or vehicle-treated mice (Figure 4 and Supplemental Figure 3). These results suggest that FTY720 treatment may affect cytokine levels during the maintenance of the lung cryptococcal granuloma.

FTY720 affects granuloma organization. To assess localization of immune cells in the lung, immunohistochemistry was performed to stain paraffin-embedded sections of lungs isolated from mice at day 60 after compound administration from the reactivation model (day 90 after infection) (Figure 5). Mice receiving FTY720 showed a reversal in the layers of granuloma structure, as F4/80-positive cells were found in a thick layer outside of the fibrotic ring of fibroblasts, which stained positive for collagen deposition via Verhoeff-Van Gieson (VVG) stain, whereas macrophages remained inside this layer for both the vehicle-treated control and BAF312 treatments (Figure 5A). All 3 groups showed comparable collagen deposition throughout the granuloma (Figure 5A) and fibroblast staining at the edges of the granuloma (Supplemental Figure 4), suggesting that the altered macrophage localization is not due to a mechanical disruption. Additional sections from granulomas taken at day 50 (matching with $H \& E$ and mucicarmine in Figure 1) also showed similar colla-

\section{Table 1. Minimum inhibitory concentration to inhibit the growth of 50\% of the tested organism was evaluated for FTY720, FTY720P, and BAF312}

MIC50, $\mu \mathrm{g} / \mathrm{mL}$

\begin{tabular}{|c|c|c|c|c|c|c|}
\hline & Cn H99 & Cn $\triangle g c s 1$ & $\mathrm{Cg}$ E)B51 & Cg R272 & Cg R265 & Ca A39 \\
\hline FTY720 & 2 & 2 & 2 & 2 & 2 & 4 \\
\hline BAF312 & 2 & 4 & 1 & 4 & 2 & $>16$ \\
\hline
\end{tabular}

MIC50, minimum inhibitory concentration for C. neoformans (Cn), C. gattii (Cg), and C. albicans (Ca). $n=3$ independent experiments. 

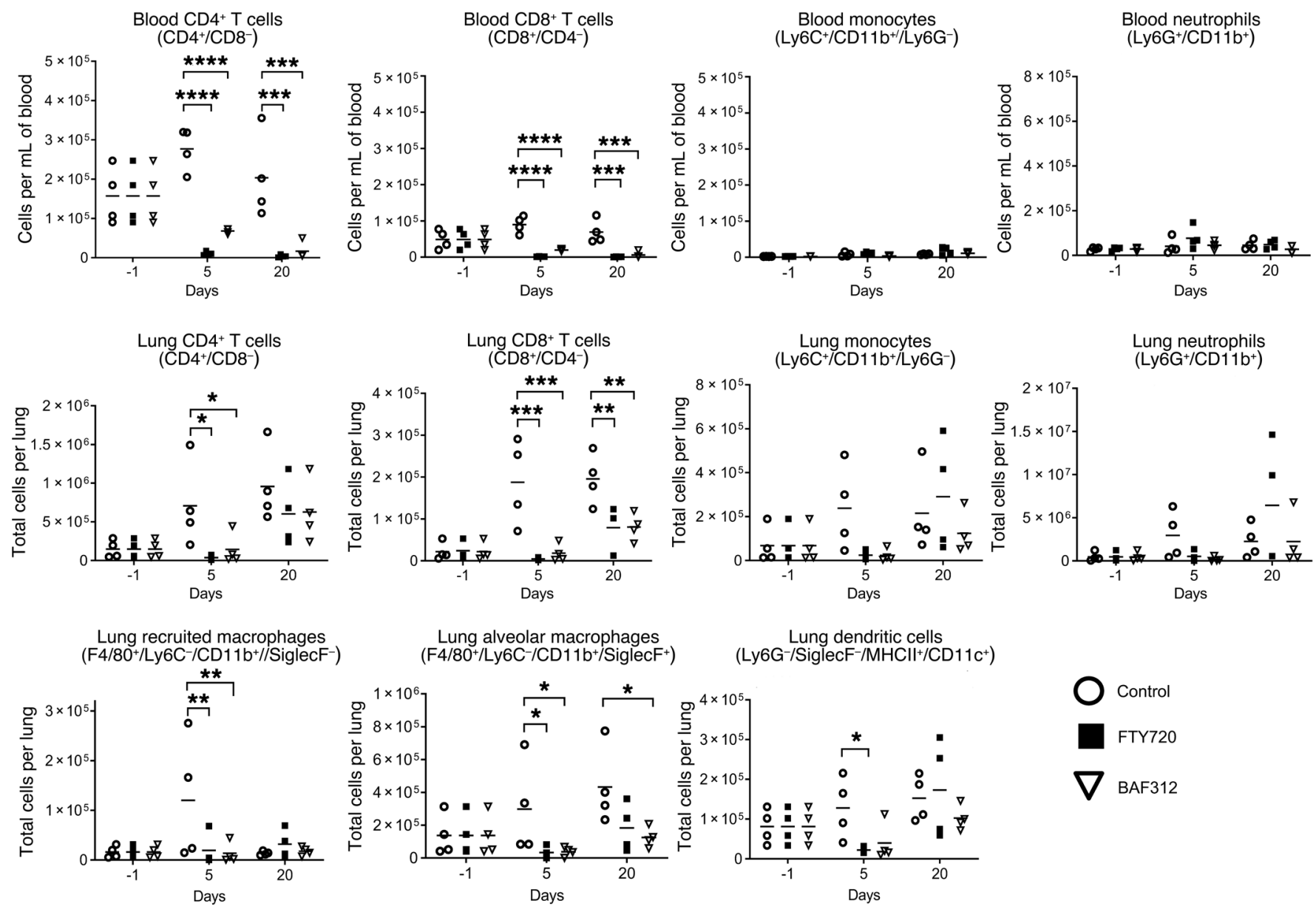

Lung dendritic cells
$\left(\right.$ Ly6G-/SiglecF-/MHCII+/CD11c $\left.{ }^{+}\right)$

Figure 3. FTY720 and BAF312 both cause a decrease in T cells and innate immune cells in the lung and blood. Mice were infected for 30 days with C. neoformans $\Delta g c s 1$ before FTY720, BAF312, or vehicle control $\left(\mathrm{H}_{2} \mathrm{O}\right)$ administration. For the blood analysis, $n=4$ mice per group at each time point and for lungs, $n=4$ mice per group at each time point (1 day before compound administration [day -1 ], day 5 , and day 20 after compound administration). Experiment was conducted 2 times. For lung samples, leukocytes were labeled intravascularly to distinguish between cells in the lung parenchyma and vasculature before staining to identify immune cell populations. Statistical significance was determined using 2-way repeated measures ANOVA with Bonferroni's multiple comparisons post hoc test. $P$ values were corrected for multiplicity using the Bonferroni's adjustment. ${ }^{*} P<0.05,{ }^{*} P<0.01,{ }^{* *} P<0.001,{ }^{*}{ }^{*} P$ $<0.0001$ compared with the control. Adjusted $P$ values available in Supplemental Table 1.

gen and elastin deposition at the edges of the granuloma across the 3 groups (Supplemental Figure 5). Using CD38 and EGR2 as markers of M1 and M2 macrophage polarization (27), it was found that both FTY720 and BAF312 treatments induced an increased M1 polarization inside the fibrotic layer but only FTY720 treatment was associated with a strong M2 phenotype within this layer compared with both vehicle-treated control and BAF312 treatments (Figure 6, A and B). Multiple granulomas showed similar patterns of CD38 and EGR2 staining (Supplemental Figure 6).CD4 and CD8 staining were found in all 3 groups and remained around the outside of the granuloma structure, suggesting that $\mathrm{T}$ cell localization at the granuloma is not a major factor for reactivation (Supplemental Figure 7).

FTY720 affects macrophage effector functions through S1P receptor 3. To examine the role of S1PR3, which is one of the receptors targeted by FTY720 but not BAF312 (Figure 7A), we tested the effect of drug treatment on phagocytosis and production of reactive oxygen species (ROS) in primary and cell line (MH-S) alveolar macrophages. We found that treatment with FTY720P significantly decreased phagocytosis of C. neoformans (Figure 7B).
FTY720 has been shown to have a differential effect on S1PR3 (Figure 7A). Previous research showed that although FTY720P agonizes S1PR3 pathways that are linked to $G$ alpha $i$ and $G$ alpha $12 / 13$ proteins, it also inhibits signaling through $G$ alpha $q(27,28)$. To study if S1PR3-G alpha q signaling could be mediating the effect on phagocytosis, compounds that target S1PR3 by either selectively agonizing (CYM5541), antagonizing (TY52156), or affecting downstream pathways of $G$ alpha $q$ (phospholipase C, U73122) were used (Figure 7A). We found that antagonism of the receptor through treatment with TY52156 or inhibition of phospholipase C with U73122 could also cause decreased phagocytosis (Figure 7C), suggesting that the impaired phagocytosis is related to inhibition of S1PR3 coupling to G alpha q. Treatment with FTY720P also led to a significant decrease in ROS production (Figure 7D). These effects of drug treatment on phagocytosis were corroborated and confirmed using additional inhibitors (e.g., Manoalide and CAY10444) in MH-S macrophages (Supplemental Figure 8).

Next, phagocytosis by S1PR3-deficient macrophages was examined. It was found that $S 1 p r 3^{-/-}$knockout macrophages had 

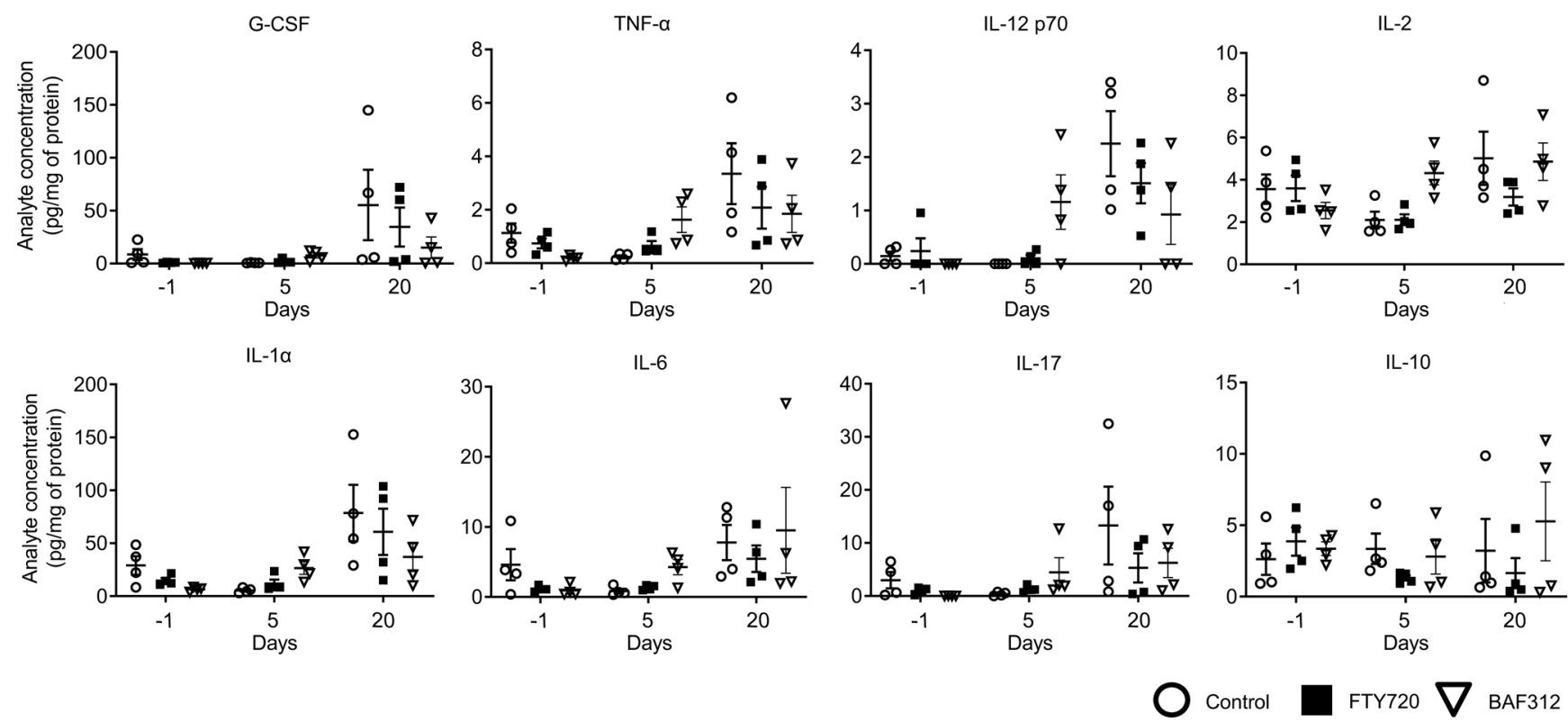

Figure 4. FTY720 does not induce a major change in lung cytokine profiles compared with BAF312. Mice were infected for 30 days with C. neoformans $\Delta g c s 1$ before FTY720, BAF312, or vehicle control $\left(\mathrm{H}_{2} \mathrm{O}\right)$ administration. For the lung analysis, $n=4$ mice per group at each time point (before compound administration [day -1], day 5, and day 20 after compound administration) were used. Cytokine levels (pg/mL) were normalized to protein concentration $(\mathrm{mg} / \mathrm{mL})$ determined by the Bradford assay. All error bars represent SEM.

impaired phagocytosis (Figure $8 \mathrm{~A}$ ), which was not rescued by addition of S1P (Figure 8B). Interestingly, S1pr3 $3^{-1}$ knockout macrophages also had a deficiency in killing activity, which similarly was not rescued by S1P supplementation (Figure 8C). Additionally, when we examined production of ROS by primary alveolar macrophages upon phagocytosis of C. neoformans, we found a significant decrease in ROS production in S1PR3-deficient macrophages (Figure 8D). These effects are not restricted to the $C$. neoformans $\triangle g c s 1$, as they were validated in C. neoformans WT H99 (Supplemental Figure 9). These results suggest that compared with BAF312, FTY720 affects specific functions of macrophages in controlling C. neoformans via phagocytosis and killing, which are essential at the granuloma site for effective containment of fungal cells within the granuloma.

\section{Discussion}

The increase of cryptococcal cases during treatment with FTY720 in patients with multiple sclerosis prompted us to study the effect of this drug and the next generation drug BAF312 in mouse models of cryptococcosis. Our data suggest that FTY720 affects the reactivation rather than the primary infection model and it does so by compromising the intracellular pathways controlled by S1PR3, an S1P receptor targeted by FTY720 but not by BAF312.

The observed decrease in survival during administration of FTY720 in the reactivation model is consistent with our past work in SK1 knockout mice, where increased lung burden was observed in the absence of S1P signaling (24). In the current study, we show that S1P signaling mediated by S1PR3 becomes critical in controlling C. neoformans in the granuloma. Despite both treatments causing a decrease in circulating T cells, only FTY720 resulted in a decrease in survival, an increase in lung burden, as well as disor- ganization and M2 polarization of macrophages within the granuloma structure. Using flow cytometry, no major differences in immune cell recruitment to the lung were observed between the 2 treated groups. Cytokine analysis in lung tissue did show some decrease in IL-2, IL-4, IL-6, IL-9, IL-10, and IL-15 at 20 days after treatment with FTY720. Although these differences were minor and not statistically significant, they may be important because IL- 4 and IL-10 are pro-M2 cytokines, whereas IL- 2 and IL- 6 are pro-M1 cytokines $(29,30)$. Among these, IL-2 and IL-6 levels in the lung tissue were more relatively abundant and thus, decreasing IL-2 and IL-6 may result in a more profound effect on M1 polarization compared with the effects of IL-4 and IL-10 on M2 polarization. Consequently, macrophages may be skewed toward M2 polarization. This is exactly what we observed in macrophages contained within the FTY720-treated granulomas. IL-15 induces $\mathrm{CD} 8^{+} \mathrm{T}$ cell-mediated killing of C.neoformans (31), and its decrease in FTY720-treated mice may contribute to the decreased cryptococcal killing at the granuloma site. IL-9 is a pleiotropic cytokine mainly associated with allergic reactions and very little is known about the role of IL-9 on C. neoformans infection or granuloma formation (32). Further studies are needed to elucidate its role on cryptococcosis. These results suggest that FTY720 may affect cytokine functions important for $C$. neoformans containment at the site of the granuloma.

Notably, the key difference between the mode of action of these compounds is the selectivity with which they bind to S1P receptors. While FTY720P binds to S1PR1, 3, 4, and 5, BAF312 only has affinity for S1PR1 and 5 at therapeutic doses $(17,20)$. The results seen in the reactivation model point to the role of S1PR3 in the maintenance of cryptococcal granulomas, as this is one of the receptors targeted by FTY720, but not BAF312, and highly expressed on macrophages. 
A
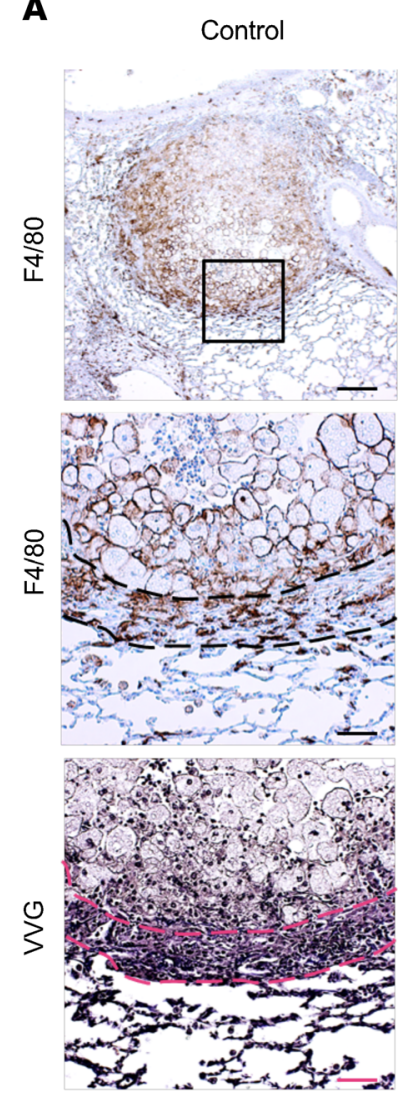

FTY720
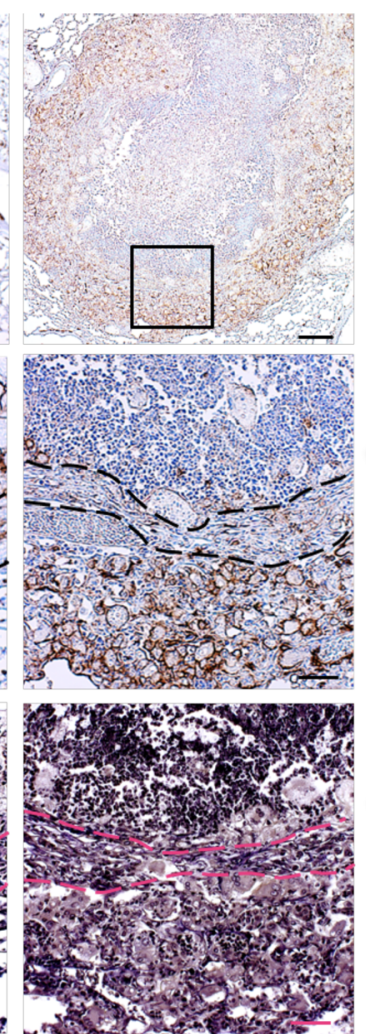

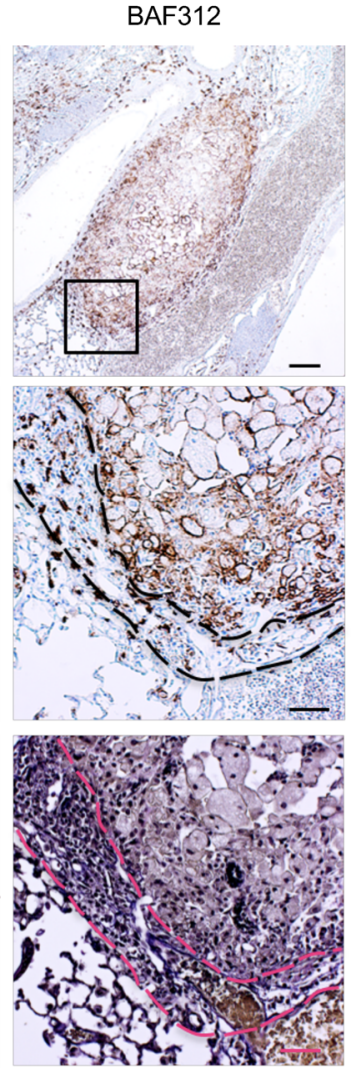

B

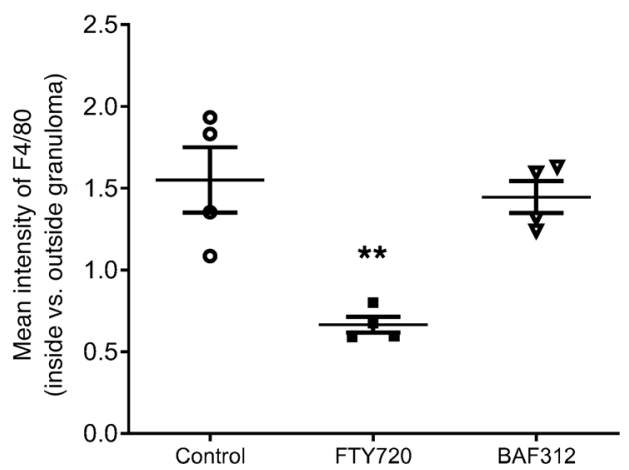

Figure 5. Treatment with FTY720 but not BAF312 affects macrophage organization in the granuloma structure. (A) Mice were infected for 30 days with C. neoformans $\triangle g c 51$ before FTY720, BAF312, or vehicle control $\left(\mathrm{H}_{2} \mathrm{O}\right)$ administration. At day 60 after compound administration lungs were processed for F4/80 immunohistochemistry and Verhoeff-Van Gieson (VVG) staining. Scale bars: $200 \mu \mathrm{m}$ (top), $50 \mu \mathrm{m}$ (middle and bottom). Black box indicates enlarged area. The dashed line indicates the fibrotic granuloma layer identified by the red collagen and black elastin staining in VVG. (B) The mean intensity of F4/80 staining inside and outside the bounds of the granuloma, as delineated by the collagen deposition seen in VVG, was quantified using Image J software, $n=4$. All error bars represent SEM. Comparisons were done with 1-way ANOVA with Bonferroni's multiple comparisons post hoc test. $P$ value was corrected for multiplicity using the Bonferroni's adjustment. ${ }^{* *} P=0.0021$ compared with control.

Macrophages have been shown to be key in controlling $C$. neoformans in immune-competent hosts, can harbor intracellular C. neoformans, and aid in dissemination when not properly activated. They can function as Trojan horses, which carry fungal cells into organs and tissues $(7,33-37)$. The central role that macrophages play in controlling cryptococcosis, particularly at the granuloma site $(35,38,39)$, in combination with our observation that they become mislocalized upon FTY720 treatment, led us to examine macrophage interactions with C. neoformans. We focused on S1PR3 and not on S1PR4 (the other S1P receptor targeted by FTY720 and not BAF312) because S1PR3 is present on macrophages (in addition to other immune cells) and has been linked to inflammation in the lung and bactericidal activity of macrophages in septic patients (40-43). Additionally, S1PR4 is unlikely to play a role since it has been shown to be downregulated in macrophages under M1-polarizing conditions; M1 polarization is known to be essential for controlling cryptococcosis (44, 45). Thus, the effect of FTY720 on the S1PR4 on macrophages would be beneficial in treating cryptococcosis.

While examining the way that FTY720 and S1PR3 affect the functions of macrophages, we found that deficiency of S1PR3 in primary alveolar macrophages led to a decrease in phagocytosis and production of ROS, both of which are key effector functions of macrophages for controlling C. neoformans. The decrease in phagocytosis and ROS production could also be seen when cells were treated with FTY720P, but not when treated with BAF312.

In order to validate these results, we examined the pathways downstream of S1PR3 using several compounds that either agonize (CYM5541), antagonize (TY52156 and CAY10444), or inhibit downstream of the G alpha q protein (U73122 and Manoalide). FTY720P has been reported to agonize S1PR3 signaling through $\mathrm{G}$ alpha $\mathrm{i}$ and $\mathrm{G}$ alpha $12 / 13$, and antagonize signaling via $G$ alpha $q(28,46)$. $G$ alpha $q$ signaling has been shown to activate phospholipase C (PLC), which has been linked to macrophage effector functions such as phagocytosis and maturation of the phagolysosome $(47,48)$, but this has never been demonstrated during an infection process. Decreased phagocytosis was observed when S1PR3 was antagonized or when PLC was inhibited, suggesting that FTY720P mediates a decrease in phagocytosis by antagonizing $G$ alpha $\mathrm{q}$, thereby decreasing PLC signaling.

Using the same set of compounds, we found that only treatment with FTY720P caused a decrease in ROS, suggesting that 
A

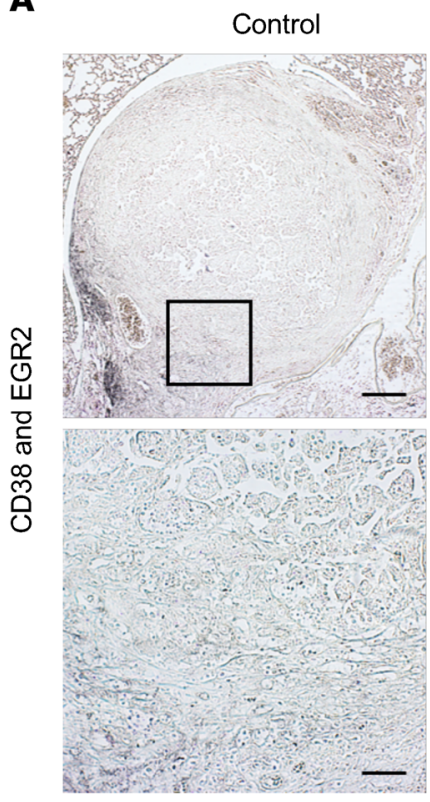

FTY720
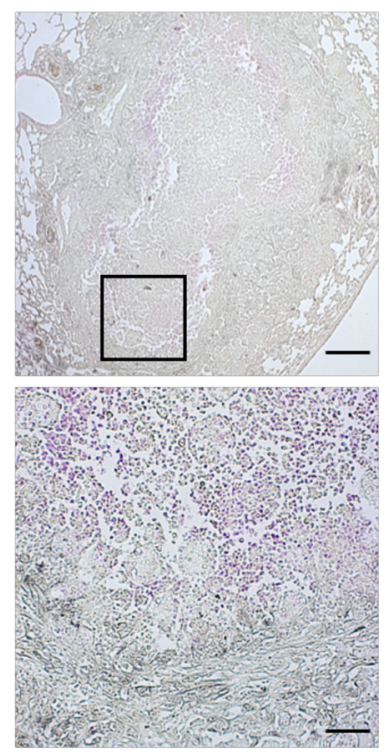

BAF312
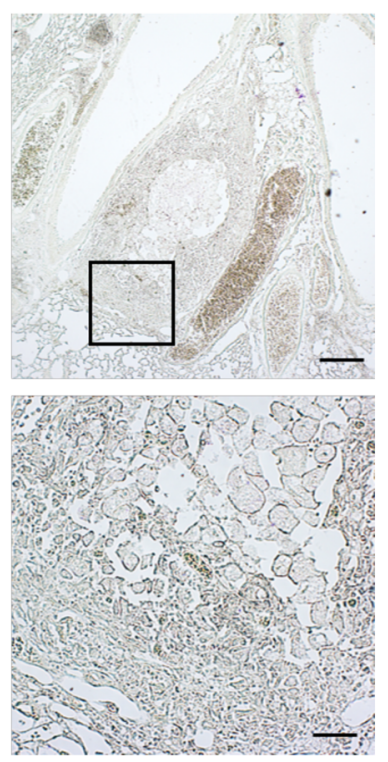

Figure 6. Treatment with FTY720 but not BAF312 affects M2 macrophage polarization in the granuloma structure. (A) Mice were infected for 30 days with $C$. neoformans $4 g c s 1$ before FTY720, BAF312, or vehicle control $\left(\mathrm{H}_{2} \mathrm{O}\right)$ administration. At day 60 after compound administration, lungs were processed for CD38 (gray) and EGR2 (magenta) immunohistochemistry to assess for M1 and M2 macrophage polarization, respectively. Scale bars: $200 \mu \mathrm{m}$ (top), $50 \mu \mathrm{m}$ (bottom). (B) The relative intensity of CD38 and ECR2 staining inside versus outside the bounds of the granuloma, as delineated by the collagen deposition seen in VVG of Figure 5, was quantified using Image J software, $n=3$. All error bars represent SEM. Comparisons were done with 2-way ANOVA with Bonferroni's multiple comparisons post hoc test. $P$ value was corrected for multiplicity using Bonferroni's adjustment. ${ }^{*} P=0.0393,{ }^{* *} P=0.0084$, ${ }_{* * * *}^{* *}<0.0001$ compared with the control. $\# \# \# P<0.0001$ compared with BAF312.

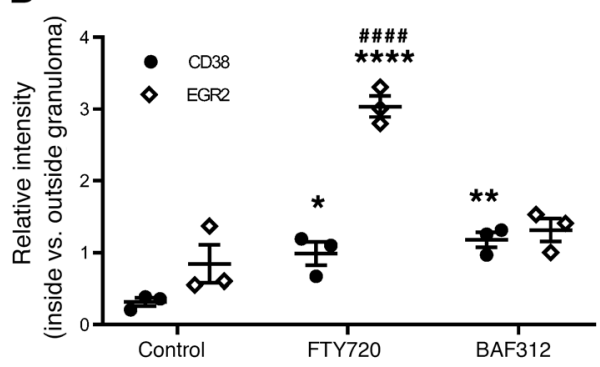

the effect is independent of pharmacological agonism or antagonism of the receptor, or inhibition of PLC. The results using our pharmacological approach validated our findings in S1PR3deficient macrophages, which were found to produce less ROS in response to C. neoformans and to exert impaired killing activity toward C. neoformans. Interestingly, killing activity could not be restored by supplementation of S1P when S1pr3 was deleted. ROS production by macrophages has been previously linked to S1PR3 signaling (43). ROS production is thought to be downstream of $\mathrm{G}$ alpha i/phosphoinositide 3-kinase (PI3K), which is opposed by $G$ alpha $12 / 13$ signaling. This regulatory effect of the downstream $G$ alpha proteins makes it difficult to ascertain how activation of both pathways by FTY720P would impact ROS production $(43,49)$.

It has also been suggested that S1P promotes M1 polarization in macrophages through S1PR3 $(50,51)$ and that FTY720 blocks this polarization and may instead promote M2 polarization (50, $52,53)$. Indeed, we observed that FTY720 treatment induces M2 polarization, which is confined within the fibrotic layer of the granulomas. Although the cytokine analysis suggested an M2polarized granulomatous response, this requires further studies focused at the site of the granuloma because our analysis was performed with the total lung parenchyma.

Our observations suggest that treatment with FTY720 but not with BAF312 interferes with S1P-S1PR3 signaling necessary for proper localization and polarization of macrophages at the cryptococcal granuloma site. Another potential explanation for the mislocalization of macrophages is that the cells are moving outside of the granuloma in response to the increase in fungal burden; the localization and effector functions could be segregated behaviors. It is also possible that S1P-S1PR3 signaling is somehow promoting macrophage retention in the granuloma, which is not promoted by FTY720P-S1PR3 signaling. Further studies are needed to evaluate these possibilities in detail.

Our results clearly show that treatment with FTY720 affects phagocytosis and ROS production in macrophages and these functions are controlled through S1PR3. Our results using the S1pr3-macrophages and BAF312 support our conclusions. Nitric oxide production was not affected by treatment with FTY720, BAF312, or by deletion of S1pr3 (data not shown).

A model of how this signaling impacts the maintenance of cryptococcal granulomas is shown in Figure 9. FTY720P binds to S1PR3 on macrophages and leads to decreased ROS production and phagocytosis by deregulating S1PR3 function and opposing $\mathrm{G}$ alpha q signaling, respectively. This leads to a loss of control of C. neoformans replication in the granuloma, particularly within macrophages, which are now predominantly found outside the fibrotic granuloma layer. The macrophages remaining within the fibrotic layer develop an M2 phenotype. Conversely, BAF312 allows for S1P to signal through S1PR3 on macrophages, which 
A

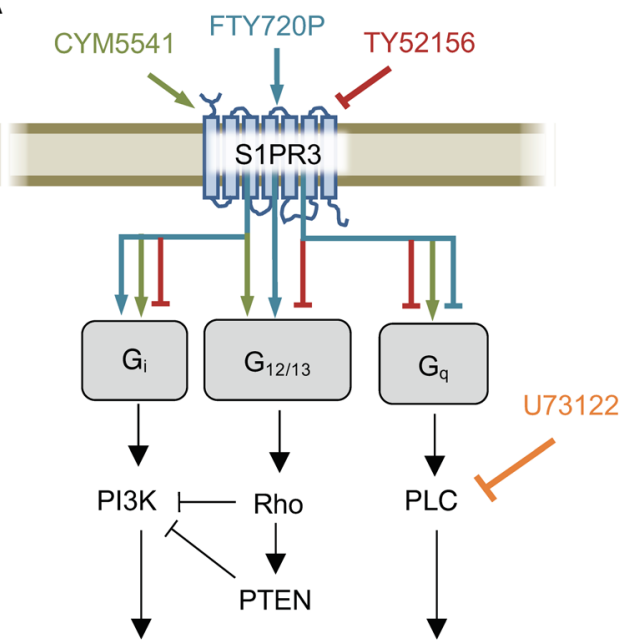

ROS/NOS?
B

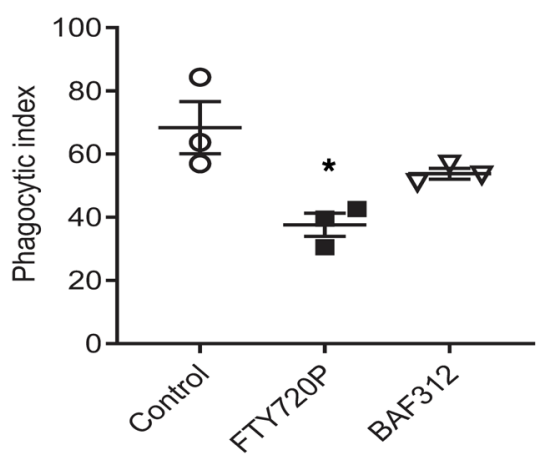

C

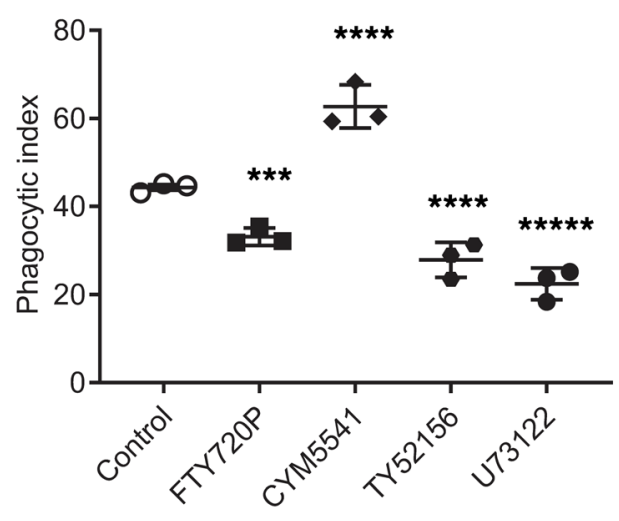

D

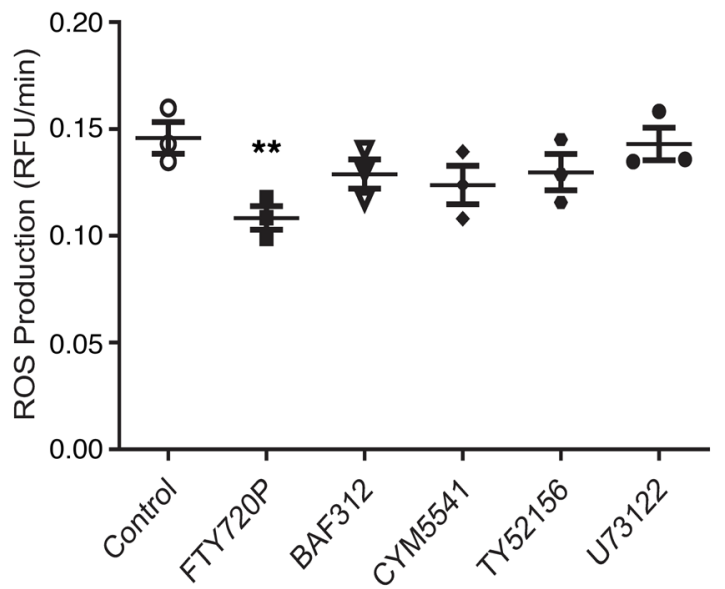

Figure 7. FTY720 impairs phagocytosis and ROS in macrophages. (A) A schematic of pathways downstream of S1PR3 in macrophages as well as the reported activity of the compounds used in $\mathbf{C}$ and $\mathbf{D}$ is shown. (B) Primary alveolar macrophages isolated from WT mice were treated overnight with $1 \mathrm{nM}$ of indicated compound, $n=3$. Cells were subsequently coincubated with opsonized $C$. neoformans $\Delta g c s 1$ and phagocytic index was calculated by microscopic observation. Experiment was conducted 3 times. (C) Primary alveolar macrophages isolated from WT mice were treated with indicated compound for 1 hour $n=3$ each. Phagocytic index was performed as in B. Experiment was conducted 3 times. (D) Primary alveolar macrophages from WT mice were treated overnight with the respective compound and analysis of ROS production was performed, $n=3$. Experiment was conducted 3 times. All error bars represent SEM and statistical comparisons were done using the 2 -sided Student's $t$ test $\left(\mathbf{B}:{ }^{*} P=0.0313\right)$ or 1-way ANOVA with Bonferroni's multiple comparisons post hoc test. $P$ values were corrected for multiplicity using the Bonferroni's adjustment $\left(\mathbf{C}:{ }^{* *} P=0.0098,{ }^{* * *} P<0.001,{ }^{* * * * *} P<0.0001 ; \mathbf{D}\right.$ : $\left.{ }^{* *} P=0.0218\right)$.

leads to phagocytosis and ROS production that keeps C. neoformans replication in check inside granulomas. Macrophages remain within the granuloma fibrotic ring and granuloma structure is preserved. Activity of BAF312 on S1PR1 or S1PR5 was not sufficient to cause reactivation in this mouse model. This mode of action of BAF312 is also supported by the data on the primary infection model using the highly virulent clinical $C$. neoformans strain H99, where mouse survival significantly improved by the administration of BAF312 $(P=0.0083)$. In this model, intact macrophage function, together with the antifungal activity of BAF312, become critical for controlling fungal replication. Furthermore, in addition to allowing S1P to coordinate its effects through S1PR3, it is possible that by blocking S1PR1 and S1PR5, BAF312 further augments this protective macrophage response. Macrophages may be more responsive to phagocytosis and killing when S1P acts selectively and exclusively through S1PR3. On the other hand, our results also suggest that the improvement in survival caused by BAF312 treatment may be due to its antifungal activity, which is retained in the animals as demonstrated by a significantly improved survival when mice were challenged with H99, the highly virulent clinical WT C. neoformans strain.

These findings offer key insights into the granulomatous response to C. neoformans and they also have important implications for the therapeutic use of compounds that target S1P signaling. Cryptococcal infections, including cases of fatal cryptococcal meningitis and disseminated cryptococcal infections, have been reported with FTY720 (Gilenya) in the postmarketing setting. Cryptococcal infections have generally occurred after approximately 2 years of Gilenya treatment, but may occur earlier. The relationship between the risk of cryptococcal infection and the 
A

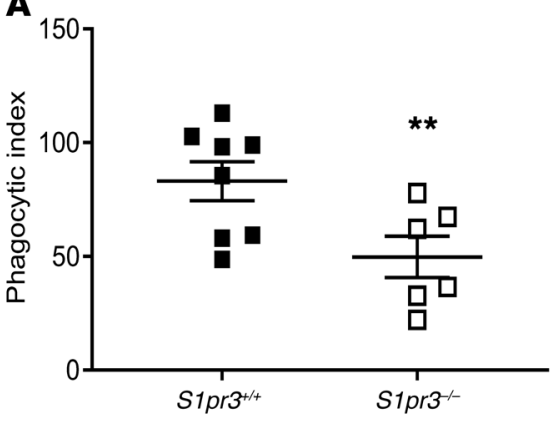

C

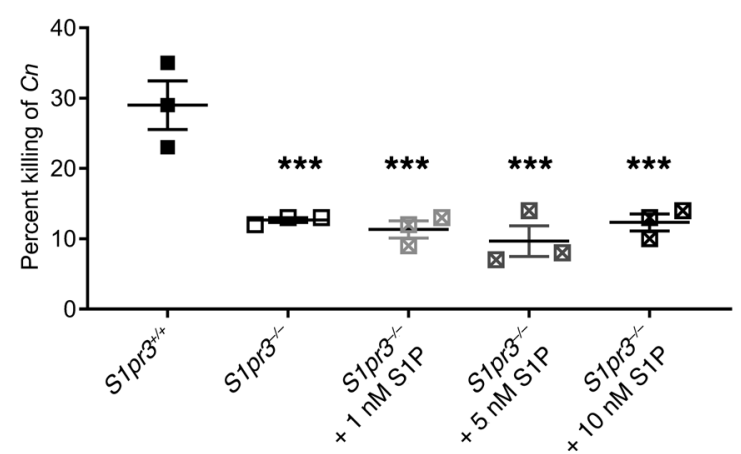

B

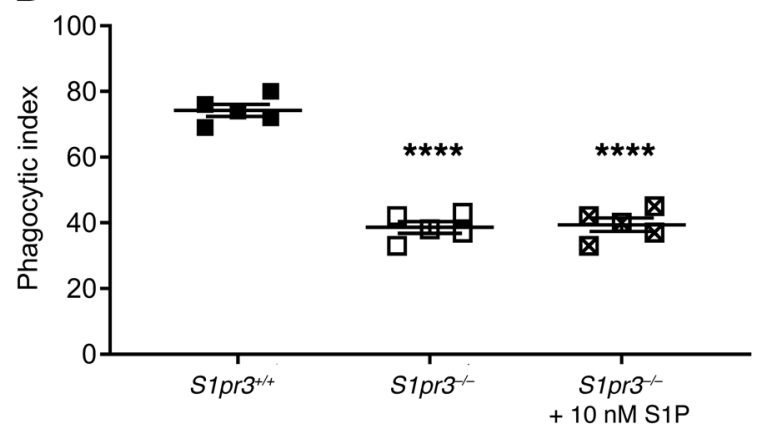

D

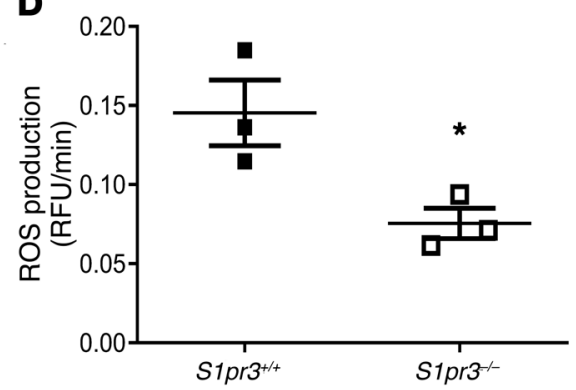

Figure 8. S1P/S1PR3 signaling is required for phagocytosis, ROS production, and intracellular killing of $C$. neoformans $\Delta g c s 1$. (A) Primary alveolar macrophages isolated from WT $(n=8)$ and S1PR3 deficient $(n=6)$ mice were coincubated with opsonized $C$. neoformans $\Delta$ gcs 1 and phagocytic index was calculated by microscopic observation. Experiment was conducted 3 times. (B) Phagocytic index for primary alveolar macrophages isolated from mice of indicated genotype ( $n=5$ each) with and without S1P supplementation were calculated as in A. Experiment was conducted 3 times. (C) Primary alveolar macrophages isolated from mice of indicated genotype $(n=3)$ with and without S1P supplementation were coincubated with opsonized $C$. neoformans $\triangle g c s 1$. After incubation, culture media were plated onto YPD agar. Percentage of killing was calculated as the difference in CFUs of $\triangle g c s 1$ incubated with or without macrophages. Experiment was conducted 5 times. (D) Primary alveolar macrophages of the indicated genotype $(n=3)$ were analyzed for ROS production after coincubation with opsonized C. neoformans $\triangle g$ cs1. Experiment was conducted 5 times. All error bars represent SEM and statistical comparisons were done using 2-sided Student's $t$ test $\left({ }^{*} P=0.0378,{ }^{* *} P=0.0222\right)$ or 1 -way ANOVA with Bonferroni's multiple comparisons post hoc test. $P$ values were corrected for multiplicity using the Bonferroni's adjustment ${ }^{* * *} P<0.001,{ }^{* * *} P<0.0001$, compared with control).

duration of treatment is currently unknown. Fungal infections, particularly cryptococcosis, are difficult to treat, and can be lifethreatening. In the published case reports, the 2 patients who did not survive likely succumbed to complications arising from C. neoformans infections (54-56). An overview of all cryptococcal cases in patients taking Gilenya is the subject of further study by this research team. The key to survival is early detection and treatment to control the infection; patients with symptoms and signs consistent with a cryptococcal infection should undergo prompt diagnostic evaluation and treatment. BAF312 (Mayzent) has been recently approved by the United States Food and Drug Administration for treatment of secondary progressive MS, but it is too early to tell if there is a reduced risk of cryptococcal meningitis.

In conclusion, we show for what we believe is the first time that FTY720 reactivates cryptococcal infection from the granuloma, explaining why several cases of cryptococcosis have been observed in MS patients taking FTY720. Our studies also address certain paradigms in the field. Lymphopenia is necessary but not sufficient to reactivate C. neoformans, as BAF312 causes a decrease in circulating $\mathrm{T}$ cells but does not lead to reactivation or increased virulence in a primary infection. Our results suggest that macrophage function must be impaired at the site of the granuloma for $C$. neoformans to escape.

\section{Methods}

Cells, media, and pharmacological compounds. C. neoformans var. grubii serotype A strain H99 (WT) and a mutant C. neoformans strain lacking GCS1 ( $\triangle g c s 1)$ (23) were used in this study. Both strains were grown in yeast nitrogenous base medium containing $2 \%$ glucose, $\mathrm{pH} 7.2$, for 16 to 18 hours at $30^{\circ} \mathrm{C}$ before experiments. Alveolar macrophage cell line MH-S (ATCC CRL-2019) and primary alveolar macrophages were cultured in complete RPMI-1640 (supplemented with 10\% FBS and $1 \%$ penicillin/streptomycin) at $37^{\circ} \mathrm{C}+5 \% \mathrm{CO}_{2}$. Both primary alveolar macrophages and MH-S cells were stimulated with 100 units of mouse IFN- $\gamma$ overnight ( 1 unit $=0.1 \mathrm{ng} / \mathrm{mL}$ ) before use. Peritoneal macrophage cell line J774.1 (ATCC TIB-67) was cultured in complete DMEM (supplemented with 10\% FBS and 1\% penicillin/streptomycin). FTY720 and BAF312 were provided by Novartis Pharma AG whereas NBDFTY720 (Cayman Chemicals), FTY720P (Echelon or Cayman Chemicals), CYM5541 (Cayman Chemicals), TY52156 (Tocris), CAY10444 (Cayman Chemicals), U73122 (Tocris), Manoalide (Santa Cruz Biotechnology), and LY294002 (Cayman Chemicals) were purchased.

In vitro susceptibility testing. Minimal inhibitory concentrations (MICs) were determined by following the methods of the Clinical and Laboratory Standards Institute (CLSI) with modifications, as previously described (57). The MICs were determined as the lowest 
A

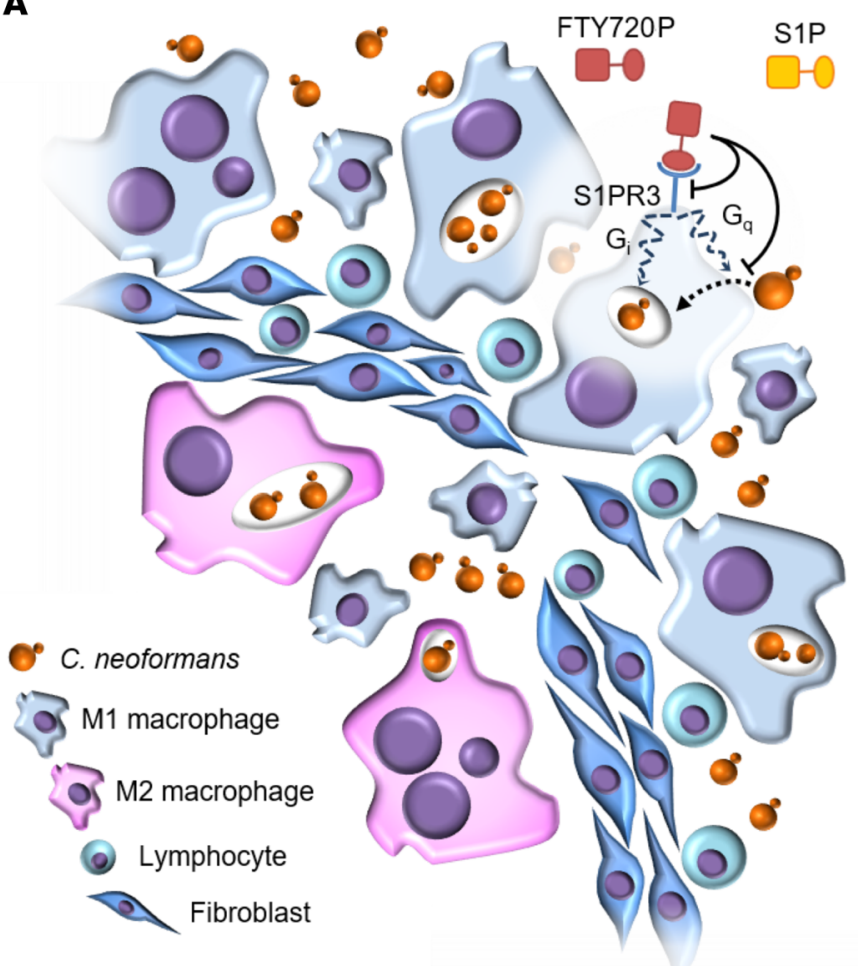

B

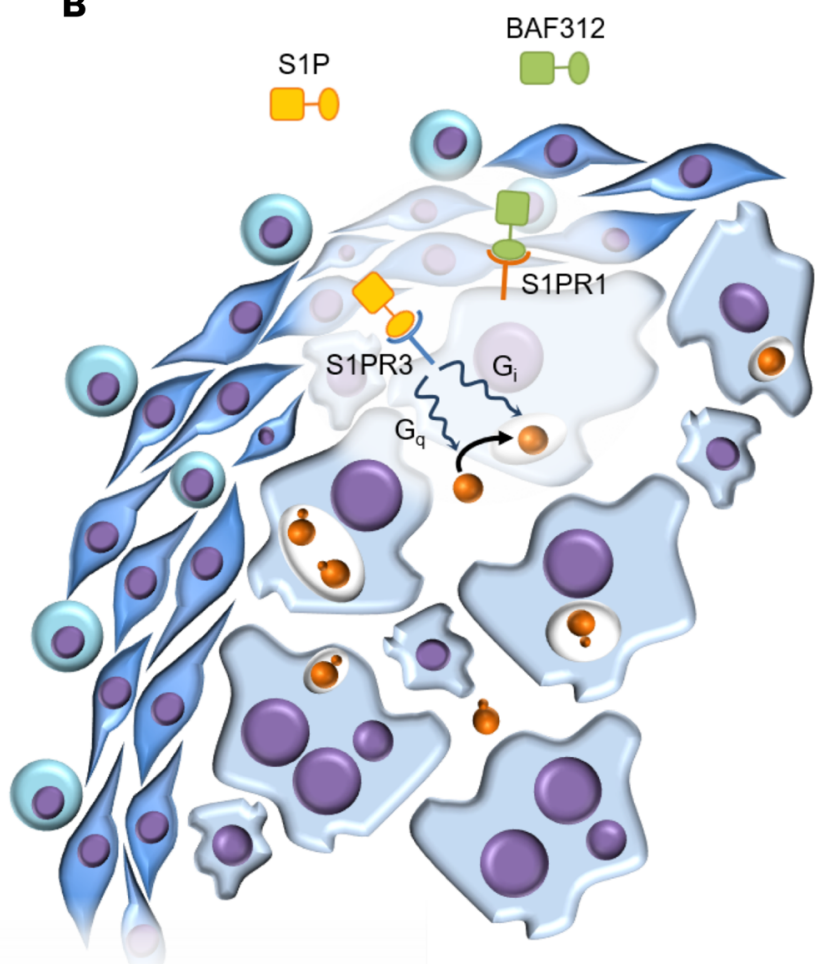

Figure 9. Model of FTY720 versus BAF312 action on cryptococcal reactivation. (A) When FTY720 is administered, the FTY720-phosphate (FTY720P) affects S1PR3 (along with S1PR1, S1PR4, and S1PR5; not illustrated). This leads to decreased phagocytosis, intracellular killing, and R0S production, which disrupt the maintenance of the granuloma. Fungal cells are able to replicate and escape to cause a reactivation. Granuloma structure is altered; macrophages are found outside of the fibrotic granuloma layer and those remaining inside the granuloma show an M2 polarization. (B) When BAF312 is administered, S1PR3 signaling is unaffected, granuloma organization is maintained, and no reactivation takes place.

concentration of the compound that inhibited $50 \%$ of growth compared with the control.

In vitro cytotoxicity assay. Cytotoxicity of pharmacological compounds were assessed via the MTT assay. MH-S cells were treated with $1 \mu \mathrm{M}$ of respective compound for 1 hour in complete RPMI-1640. After incubation with compound, media was removed and $50 \mu \mathrm{L}$ of $5-\mathrm{mg} / \mathrm{mL}$ 3-(4,5-dimethylthiazol-2-yl)-2,5-diphenyltetrazoliumbromide (MTT) solution in 1X PBS was added to each well (Thermo Fisher Scientific). The plates were incubated for an additional 4 hours at $37^{\circ} \mathrm{C}+5 \% \mathrm{CO}_{2}$. The formazan crystal formed inside the cell was dissolved by adding $100 \mu \mathrm{L}$ DMSO. The optical density was measured at $570 \mathrm{~nm}$ using the SpectraMax M5 microplate reader (Molecular Devices).

Thin layer chromatography. NBD-FTY720 (Cayman Chemicals) was added to the media of either mammalian cells (J774A.1) or C. neoformans cells ( $C n$ H99 and $C n \Delta g c s 1$ ) at a concentration of $2 \mu \mathrm{g} / \mathrm{mL}$ and incubated for 1 hour at $37^{\circ} \mathrm{C}$ with $5 \% \mathrm{CO}_{2}$. NBD-FTY720 was extracted from the media as previously described (17) with 2 volumes of 1:2 chloroform/methanol. Extracted lipids were dried using a nitrogen evaporator (N-EVAP; Organomation) and the pellet was resuspended in chloroform. The lipids were separated on a thin layer chromatography silica gel glass plate (MilliporeSigma) using 80:10:1 (vol/vol/vol) chloroform/ methanol/water and fluorescence was visualized using the GelDoc-IT (UVP).

Mouse strains. CBA/J female and male mice obtained from Envigo aged 3 to 4 weeks were used for all survival, tissue burden, flow cytometry, and histological studies. S1PR3-deficient mice (S1pr3 ${ }^{-/-}$), generated previously at NIH by Kono et al. (58), were shared with us with permission of Richard Proia (NIH, Bethesda, Maryland, USA) from the colony at Wayne State University by Menq-Jer Lee (Wayne State University, Detroit, Michigan, USA). Heterozygotes were housed and bred at the animal facility at Stony Brook University to produce homozygous offspring. Mice were genotyped using polymerase chain reactions with the following primers: (5'-TCAGTATCTTCACCGCCATT-3'， 5'-AATCACTACGGTCCGCAGAA-3'， 5'-GTGCAATCCATCTTGTTCAAT- ${ }^{\prime}$ ). WT alleles produce a $130 \mathrm{bp}$ fragment and the knockout produces a $380 \mathrm{bp}$ fragment that can be separated using gel electrophoresis. For all experiments, $S 1 \mathrm{pr}^{-/-}$mice were age and sex matched with WT C57BL/6 mice.

Mouse studies. Mice were fed ad libitum and monitored twice a day for discomfort and signs of disease. Endpoint criteria were defined as moribund appearance and/or significant weight loss (>20\% of the vehicle-treated control). Euthanasia was performed with $\mathrm{CO}_{2}$ asphyxiation with $100 \% \mathrm{FiCO}_{2}$ for 2 minutes, followed by cervical dislocation. For infection, mice were anesthetized with an intraperitoneal injection of $60 \mu \mathrm{L}$ xylazine/ketamine mixture containing $95 \mathrm{mg}$ ketamine and $5 \mathrm{mg}$ xylazine per $\mathrm{kg}$ of body weight. Mice were infected intranasally with $5 \times 10^{5}$ cells $/ 20 \mu \mathrm{L}$ of C. neoformans $\mathrm{H} 99$ or $\Delta g c s 1$. Each mouse received a daily gavage of compound starting at either 30 days after infection (Figure 1) or 2 days before infection (Supplemental Figure 1 and Figure 2). Compounds were given at a dose of $1 \mathrm{mg} / \mathrm{kg}$ body weight dissolved in deionized water. Vehicle-treated control mice received deionized water alone. Tissue burden analysis was performed by counting CFUs at the end of the survival study or when a mouse was euthanized due to meeting endpoint criteria. Lung and brain were 
excised and homogenized in $10 \mathrm{~mL}$ of $1 \mathrm{X}$ PBS using Stomacher 80 (Seward, United Kingdom) for 2 minutes at high speed. A quantity of $100 \mu \mathrm{L}$ homogenized sample at multiple 10 -fold dilutions were plated in duplicate onto YPD agar plates. Plates containing 30 to 250 colonies were identified and counted. Total number of CFUs per organ was calculated by adjusting for a final volume of $10 \mathrm{~mL}$ per organ and the associated dilution factor. For histological analysis, mice were euthanized at the indicated time point in the figure. Lungs were removed, thoroughly washed with $1 \mathrm{X}$ PBS and fixed in 10\% formalin solution, dehydrated in ethanol, infiltrated with paraffin, and sliced and stained with $\mathrm{H} \& \mathrm{E}$ or mucicarmine as indicated in the figure legend (Figure 1).

Flow cytometry. Flow cytometry analysis was performed on the blood and lungs of mice at the indicated time points. Three minutes before euthanasia, antibody against CD45 (clone 30-F11; BioLegend) conjugated to BV650 was injected through the tail vein to differentiate between tissue and circulating leukocytes using a previously described intravascular staining method (59). Immediately upon euthanasia, blood was taken by cardiac puncture and collected in EDTA coated vacutainers (Becton Dickinson) to prevent clotting. Lungs were then excised, washed, minced, and digested for 1 hour with $150 \mathrm{U} / \mathrm{mL}$ collagenase and $10 \mathrm{U} / \mathrm{mL}$ DNAse I in 1X PBS supplemented with $2 \%$ FBS, and pushed through a $70-\mu$ m nylon cell strainer to create a single-cell suspension. Red blood cells were removed from both blood and lung samples using ammonium-chloridepotassium (ACK) lysing buffer $\left(150 \mathrm{mM} \mathrm{NH}_{4} \mathrm{Cl}, 10 \mathrm{mM} \mathrm{KHCO}_{3}, 0.1\right.$ $\mathrm{mM} \mathrm{Na}{ }_{2}$ EDTA). Single-cell suspensions of blood and lung were then filtered through a $70-\mu \mathrm{m}$ nylon cell strainer and stained with a mix of antibodies for the following markers directly conjugated to the following fluorophores: Ly6G-PE (clone 1A8), Ly6C-APC/Cy7 (clone HK1.4), CD11b-BV510 (clone M1/70), CD11c-PE/Cy7 (clone N418), CD45-BV711 (clone 30-F11), F4/80-APC (clone BM8), MHC II-FITC (clone M5/114.15.2), CD8a-BV785 (clone 53-6.7), CD4-BV605 (clone GK1.5) from BioLegend, and Siglec F-PE/CF594 (clone E50-2440) from BD Biosciences. Cells were also stained for viability using Alexa Fluor 700 carboxylic acid, succinimidyl ester (Thermo Fisher Scientific). Labeled cell suspensions were analyzed using a LSRFortessa (Becton Dickinson) and FlowJo Software Version 10.6.2 to identify

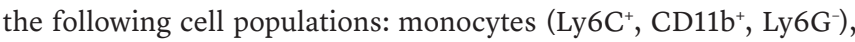
recruited macrophages $\left(\mathrm{F} 4 / 80^{+}\right.$, Ly6 $\mathrm{C}^{-}, \mathrm{CD}_{11 \mathrm{~b}^{+}}$, SiglecF- $\mathrm{F}^{-}$, alveolar macrophages $\left(\mathrm{F} 4 / 80^{+}, \mathrm{Ly} 6 \mathrm{C}^{-}, \mathrm{CD}_{11 \mathrm{~b}^{+}}\right.$, SiglecF $\left.\mathrm{F}^{+}\right)$, dendritic cells

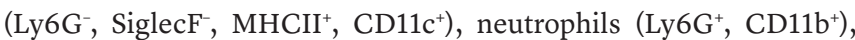
$\mathrm{CD}^{+}{ }^{+} \mathrm{T}$ cells $\left(\mathrm{CD} 4^{+}, \mathrm{CD} 8^{-}\right)$, and $\mathrm{CD}^{+} \mathrm{T}$ cells $\left(\mathrm{CD} 8^{+}, \mathrm{CD} 4^{-}\right)$. Lung populations were gated for intravascular negative staining populations. Cell populations are given either per volume of blood or calculated with respect to the total number of cells isolated from the lung as determined by an automated cell counter upon organ processing.

Immunohistochemistry and Verhoeff-Van Gieson stain. Immunohistochemistry was performed by adapting published protocols for labeling immune cells $(60,61)$. At day 60 after compound administration (day 90 after infection) mice were euthanized by $\mathrm{CO}_{2}$ inhalation and lungs were perfused with IHC Zinc Fixative solution (BD Pharmingen), dehydrated in ethanol, infiltrated, and embedded with paraffin wax. For staining, $5-\mu \mathrm{m}$ sections were used. For single label immunohistochemistry, FSP-1 (also known as S100A4; clone D9F9D, Cell Signaling) (62), F4/80 (clone D2S9R, Cell Signaling), CD4 (clone EPR19514, Abcam), or CD8 $\alpha$ (clone EPR20305, Abcam) rabbit anti-mouse monoclonal primary antibody and goat anti-rab- bit biotinylated secondary antibody (Vector Labs) were used to label sections. Antigen detection was done using the avidin-biotin-complex conjugated to horseradish peroxidase with DAB as the substrate (Vector Labs) and counterstained with Mayer's hematoxylin (SigmaAldrich). Antigens are stained brown whereas nuclei are stained blue. To assess M1/M2 polarization, rabbit anti-mouse monoclonal primary antibody for CD38 (clone EPR21079, Abcam) and goat polyclonal primary antibody for EGR2 (ab63943, Abcam) were used (27). Antigen detection was performed using horse anti-rabbit polymerized secondary conjugated to peroxidase with ImmPACT SG peroxidase substrate (Vector Labs) and horse anti-goat polymerized secondary antibody conjugated to alkaline phosphatase with ImmPACT Red phosphatase substrate (Vector Labs). CD $38^{+}$cells are stained gray whereas EGR2 ${ }^{+}$cells are stained magenta. Additional sections taken from these organs and formalin-fixed organs were stained using the Verhoeff-Van Gieson technique. Elastic fibers are stained black whereas collagen is stained pink to red. Nuclei are stained blue-black and other tissue elements (i.e., muscle, red blood cells) are stained yellow (63). Micrographs were taken using Observer.D1 microscope with AxioCam 105 color camera for taking images. Mean intensity of the DAB as well as ImmPACT SG and ImmPACT Red were quantified using Image J software.

Cytokine analysis. Cytokine analysis was performed on the lungs at the indicated time points. Lungs were excised and homogenized in $5 \mathrm{~mL}$ of $1 \mathrm{X}$ PBS using Stomacher 80 (Seward, United Kingdom) for 2 minutes at high speed and pushed through a $70-\mu \mathrm{m}$ nylon cell strainer to remove any remaining tissue debris. Cytokines in the lung tissue were analyzed using a Milliplex Mouse Cytokine/Chemokine Magnetic Bead Premixed 25 Plex kit (MilliporeSigma), following the manufacturer's overnight incubation protocol and using $1 \mathrm{X}$ PBS as the matrix for the samples. The samples were immediately run on a Bioplex 200 system running Bioplex Manager Software Version 4.1.1. Cytokine measurements $(\mathrm{pg} / \mathrm{mL})$ were normalized to protein concentration $(\mathrm{mg} / \mathrm{mL})$ as determined by the Bradford assay.

Isolation and cell culture of alveolar macrophages. Primary alveolar macrophages were isolated from the lungs via bronchoalveolar lavage fluid of S1pr3 $3^{--}$and C57BL/6 mice using $5 \times 1 \mathrm{~mL}$ sterile $1 \mathrm{X}$ PBS supplemented with $2 \% \mathrm{FBS}$. BAL fluid was subjected to centrifugation at $500 \mathrm{~g}$ for 10 minutes at $4^{\circ} \mathrm{C}$. Cell pellets were resuspended in complete RPMI-1640 and cell number was determined using a hemocytometer. Cells were plated and stimulated with 100 units of mouse IFN- $\gamma(1$ unit $=0.1 \mathrm{ng} / \mathrm{mL})$ overnight at $37^{\circ} \mathrm{C}+5 \% \mathrm{CO}_{2}$.

In vitro phagocytosis assay. Alveolar macrophages were plated and stimulated overnight as described above. C. neoformans $\mathrm{H} 99$ or $\Delta g c s 1$ was opsonized for 30 minutes at $37^{\circ} \mathrm{C}+5 \% \mathrm{CO}_{2}$ with $1 \mu \mathrm{g}$ of anti-glucoxylomannan (GXM) antibody (clone 18B7) (64) per $10^{5} \mathrm{C}$. neoformans cells in $100 \mu \mathrm{L}$ of complete RPMI-1640 before coincubation with macrophage monolayers. The anti-GXM antibody was provided by Arturo Casadevall at Johns Hopkins University (Baltimore, Maryland, USA). For experiments with primary alveolar macrophages, the antibody opsonized C. neoformans cells were added to washed macrophage monolayers at a 1:1 ratio along with lipopolysaccharide $(3 \mu \mathrm{g} / \mathrm{mL})$ and 100 units of mouse IFN- $\gamma$. C. neoformans and macrophages were allowed to interact for 2 hours at $37^{\circ} \mathrm{C}+5 \%$ $\mathrm{CO}_{2}$. Macrophage monolayers were then washed to remove extracellular C. neoformans, fixed with methanol, and stained with Giemsa to calculate phagocytic index. For experiments with MH-S cells, anti- 
body opsonized C. neoformans cells as described above was added to washed monolayers at a 1:1 ratio and allowed to interact for 2 hours at $37^{\circ} \mathrm{C}+5 \% \mathrm{CO}_{2}$. Cells were then fixed with methanol and stained with Giemsa. For experiments where compound is used, primary alveolar macrophages and $\mathrm{MH}-\mathrm{S}$ cells were exposed to the indicated compound at a concentration of $1 \mathrm{nM}$ overnight or $1 \mu \mathrm{M}$ for 1 hour, as indicated in Figure 7 and Supplemental Figure 8. Phagocytic index was calculated by microscopic observation. Phagocytic index is calculated as the percentage of yeast cells ingested per number of macrophages per field, as previously described (65). Ingested C. neoformans cells were distinguished from attached cells by the stained macrophage cell membrane surrounding the cryptococcal capsule. For each experiment, at least 4 fields were observed in at least 3 different wells per condition.

Reactive oxygen species production. ROS production was assayed using the Cellular ROS Assay Kit (ab186027, Abcam). Primary alveolar macrophages were isolated and stimulated overnight as described above. Monolayers were stained with ROS Red reagent for 1 hour per manufacturer's protocol and allowed to interact with antibody opsonized C. neoformans $\Delta g c s 1$ at a 1:1 ratio. Relative fluorescence was measured at 520 excitation /605 emission using the SpectraMax M5 microplate reader (Molecular Devices) after 30 minutes of incubation at $37^{\circ} \mathrm{C}+5 \% \mathrm{CO}_{2}$. For experiments where compound is used, alveolar macrophages were treated overnight with the respective compound at a concentration of $1 \mu \mathrm{M}$. ROS production was calculated as the difference in raw fluorescence units over time (RFU/min).

Intracellular killing assay. Primary alveolar macrophages were isolated and plated as described above. Intracellular killing was assayed by adapting a previously described method (25). Briefly, macrophages were incubated with opsonized C. neoformans $\mathrm{H} 99$ or $\Delta g c s 1$ at a 1:1 ratio for 4 hours at $37^{\circ} \mathrm{C}+5 \% \mathrm{CO}_{2}$. At the end of the incubation, the cultures were serially diluted, streaked onto YPD agar, and incubated for 48 hours at $30^{\circ} \mathrm{C}$. The numbers of CFUs were counted to assess the killing of C. neoformans compared with that of control cultures of C. neoformans alone with no macrophages. At 2 hours after infection, extracellular S1P at nanomolar concentrations dissolved in methanol was added to the macrophages. The results are reported as the percentage of killed cells compared with the number of C. neoformans cells not exposed to macrophages or S1P.

Statistics. For all statistical analysis, GraphPad Prism Version 7 software was used. Data are represented as mean \pm SEM unless noted otherwise. $\alpha$-level (type 1 error) was set at 0.05. Differences were considered significant when the probability of type 1 error was less than $5 \%(P<0.05)$. Log-rank (Mantel-Cox) test was used to compare the survival curves. For organ burden (CFU analysis), the 1-way analysis of variance (ANOVA) with Bonferroni's multiple comparisons post hoc test was used on log-transformed data. For flow cytometry data, 2-way repeated measures ANOVA with Bonferroni's multiple comparisons post hoc test was used. $P$ values are reported in Supplemental Table 1. For immunohistochemistry data, 1-way ANOVA with Bonferroni's multiple comparisons post hoc test (F4/80 and FSP-1 staining) or 2-way ANOVA with Bonferroni's multiple comparisons post hoc test (CD38/EGR2 staining) was used. For phagocytosis and ROS assays, a 2-sided Student's $t$ test or 1-way ANOVA with Bonferroni's multiple comparisons post hoc test were used where indicated. All $P$ values from multiple comparisons post hoc tests were corrected for multiplicity using the Bonferroni's adjustments.

Study approval. All animal procedures were approved by the Stony Brook University Institutional Animal Care and Use Committee (study number 341888; IACUC number 2012-1967), followed the guidelines of American Veterinary Medical Association and were in compliance with the United States Animal Welfare Act (Public Law 98-198).

\section{Author contributions}

AMB and JKY conceptualized and performed the experiments, collected the data, performed statistical analysis, and wrote the manuscript. TM helped with macrophage experiments. CL helped with in vitro antifungal activity experiments. ZQ helped with flow cytometry experiments. BS, BNH, and MDP conceptualized the experiments, discussed the data, and edited the manuscript. The order of co-first authors was determined alphabetically by last name.

\section{Acknowledgments}

We would like to acknowledge the technical support provided by the Department of Pathology, Research Histology Core Laboratory, Research Flow Cytometry Laboratory, and the Division of Laboratory Animal Resources at Stony Brook Medicine. We especially thank Kenneth R. Shroyer, Yan Ji, and Todd Rueb. We acknowledge Menq-Jer Lee for providing us with $S 1 p r 3^{-/-}$mice with permission of Richard Proia, and Arturo Casadevall for providing the anti-GXM antibody clone 18B7 used for opsonization of C. neoformans. We also acknowledge the tremendous support of Ashley Snider, Leiqing Zhang, Valentina Viscardi, and Laurie Levine for assistance with mice breeding and development of genotyping protocols. We would also like to acknowledge the contributions of Paul A. Smith for his insightful discussion and set up of the initial experiments. Finally, we acknowledge Dmitri Gnatenko of the Proteomics Core at Stony Brook Medicine for his support in performing cytokine analysis.

This work was supported by NIH grants AI136934, AI116420, and AI125770, by Merit Review Grant I01BX002924 from the Veterans Affairs Program, and by Novartis grant 73449-1-1129287 to MDP. MDP is a Burroughs Welcome Investigator in Infectious Diseases.

Address correspondence to: Maurizio Del Poeta, Department of Microbiology and Immunology, Division of Infectious Diseases, Stony Brook University, 150 Life Science Building, Stony Brook, New York 11794, USA. Phone: 631.632.4024; Email: maurizio.delpoeta@stonybrook.edu.
1. Perfect JR, Casadevall A. Cryptococcosis. Infect Dis Clin North Am. 2002;16(4):837-874.

2. Casadevall A, Coelho C, Alanio A. Mechanisms of Cryptococcus neoformans-mediated host damage. Front Immunol. 2018;9:855.

3. Rajasingham R, et al. Global burden of dis- ease of HIV-associated cryptococcal meningitis: an updated analysis. Lancet Infect Dis. 2017;17(8):873-881.

4. Pyrgos V, Seitz AE, Steiner CA, Prevots DR, Williamson PR. Epidemiology of cryptococcal meningitis in the US: 1997-2009. PLOS ONE. 2013;8(2):e56269.

5. Grebenciucova E, Reder AT, Bernard JT. Immunologic mechanisms of fingolimod and the role of immunosenescence in the risk of cryptococcal infection: A case report and review of literature. Mult Scler Relat Disord. 2016;9:158-162. 
6. Goldman DL, Lee SC, Mednick AJ, Montella L, Casadevall A. Persistent Cryptococcus neoformans pulmonary infection in the rat is associated with intracellular parasitism, decreased inducible nitric oxide synthase expression, and altered antibody responsiveness to cryptococcal polysaccharide. Infect Immun. 2000;68(2):832-838.

7. McQuiston TJ, Williamson PR. Paradoxical roles of alveolar macrophages in the host response to Cryptococcus neoformans. J Infect Chemother. 2012;18(1):1-9.

8. Saha DC, et al. Serologic evidence for reactivation of cryptococcosis in solid-organ transplant recipients. Clin Vaccine Immunol. 2007;14(12):1550-1554.

9. Novartis Pharma AG. Gilenya (fingolimod) [package insert]. U.S. Food and Drug Administration website. https://www.accessdata.fda.gov/ drugsatfda_docs/label/2012/022527s008lbl.pdf. Revised April 2012. Accessed December 20, 2019.

10. Obinata H, Hla T. Sphingosine 1-phosphate in coagulation and inflammation. Semin Immunopathol. 2012;34(1):73-91.

11. Maceyka M, Spiegel S. Sphingolipid metabolites in inflammatory disease. Nature. 2014;510(7503):58-67.

12. Bryan AM, Del Poeta M. Sphingosine-1-phosphate receptors and innate immunity. Cell Microbiol. 2018;20(5):e12836.

13. Mandala S, et al. Alteration of lymphocyte trafficking by sphingosine-1-phosphate receptor agonists. Science. 2002;296(5566):346-349.

14. Payne SG, Milstien S, Barbour SE, Spiegel S. Modulation of adaptive immune responses by sphingosine-1-phosphate. Semin Cell Dev Biol. 2004;15(5):521-527.

15. Pyne S, Pyne NJ. Translational aspects of sphingosine 1-phosphate biology. Trends Mol Med. 2011;17(8):463-472.

16. Camm J, Hla T, Bakshi R, Brinkmann V. Cardiac and vascular effects of fingolimod: mechanistic basis and clinical implications. Am Heart J. 2014;168(5):632-644.

17. Brinkmann V, et al. The immune modulator FTY720 targets sphingosine 1-phosphate receptors. J Biol Chem. 2002;277(24):21453-21457.

18. Brinkmann V, et al. Fingolimod (FTY720): discovery and development of an oral drug to treat multiple sclerosis. Nat Rev Drug Discov. 2010;9(11):883-897.

19. Matloubian M, et al. Lymphocyte egress from thymus and peripheral lymphoid organs is dependent on $\mathrm{S} 1 \mathrm{P}$ receptor 1. Nature. 2004;427(6972):355-360.

20. Gergely P, et al. The selective sphingosine 1-phosphate receptor modulator BAF312 redirects lymphocyte distribution and has species-specific effects on heart rate. $\mathrm{Br} J$ Pharmacol. 2012;167(5):1035-1047.

21. Pan S, et al. Discovery of BAF312 (Siponimod), a potent and selective S1P receptor modulator. ACS Med Chem Lett. 2013;4(3):333-337.

22. Lewis ND, et al. Circulating monocytes are reduced by sphingosine-1-phosphate receptor modulators independently of S1P3. J Immunol. 2013;190(7):3533-3540.

23. Rittershaus PC, et al. Glucosylceramide synthase is an essential regulator of pathogenic- ity of Cryptococcus neoformans. J Clin Invest. 2006;116(6):1651-1659.

24. McQuiston T, Luberto C, Del Poeta M. Role of host sphingosine kinase 1 in the lung response against Cryptococcosis. Infect Immun. 2010;78(5):2342-2352.

25. Farnoud AM, Bryan AM, Kechichian T, Luberto C, Del Poeta M. The granuloma response controlling Cryptococcosis in mice depends on the sphingosine kinase 1-sphingosine 1-phosphate pathway. Infect Immun . 2015;83(7):2705-2713.

26. McQuiston T, Luberto C, Del Poeta M. Role of sphingosine-1-phosphate (S1P) and S1P receptor 2 in the phagocytosis of Cryptococcus neoformans by alveolar macrophages. Microbiology (Reading, Engl). 2011;157(Pt 5):1416-1427.

27. Jablonski KA, et al. Novel markers to delineate murine M1 and M2 macrophages. PLoS ONE. 2015;10(12):e0145342.

28. Sensken SC, Stäubert C, Keul P, Levkau B, Schöneberg T, Gräler MH. Selective activation of $G$ alpha i mediated signalling of S1P3 by FTY720-phosphate. Cell Signal. 2008;20(6):1125-1133.

29. Chen GH, McNamara DA, Hernandez Y, Huffnagle GB, Toews GB, Olszewski MA. Inheritance of immune polarization patterns is linked to resistance versus susceptibility to Cryptococcus neoformans in a mouse model. Infect Immun. 2008;76(6):2379-2391.

30. Wang J, Zeng Y, Luo W, Xie X, Li S. The role of Cryptococcus in the immune system of pulmonary Cryptococcosis patients. PLOS ONE. 2015;10(12):e0144427.

31. Ma LL, et al. CD8 T cell-mediated killing of Cryptococcus neoformans requires granulysin and is dependent on CD4 T cells and IL-15. J Immunol. 2002;169(10):5787-5795.

32. Goswami R, Kaplan MH. A brief history of IL-9. J Immunol. 2011;186(6):3283-3288.

33. Osterholzer JJ, Milam JE, Chen GH, Toews GB, Huffnagle GB, Olszewski MA. Role of dendritic cells and alveolar macrophages in regulating early host defense against pulmonary infection with Cryptococcus neoformans. Infect Immun. 2009;77(9):3749-3758.

34. Shao X, Mednick A, Alvarez M, van Rooijen N, Casadevall A, Goldman DL. An innate immune system cell is a major determinant of speciesrelated susceptibility differences to fungal pneumonia. J Immunol. 2005;175(5):3244-3251.

35. Kechichian TB, Shea J, Del Poeta M. Depletion of alveolar macrophages decreases the dissemination of a glucosylceramide-deficient mutant of Cryptococcus neoformans in immunodeficient mice. Infect Immun. 2007;75(10):4792-4798.

36. Herring AC, Lee J, McDonald RA, Toews GB, Huffnagle GB. Induction of interleukin-12 and gamma interferon requires tumor necrosis factor alpha for protective T1-cell-mediated immunity to pulmonary Cryptococcus neoformans infection. Infect Immun. 2002;70(6):2959-2964.

37. Charlier C, Nielsen K, Daou S, Brigitte M, Chretien F, Dromer F. Evidence of a role for monocytes in dissemination and brain invasion by Cryptococcus neoformans. Infect Immun. 2009;77(1):120-127.

38. Goldman D, Lee SC, Casadevall A. Pathogenesis of pulmonary Cryptococcus neoformans infection in the rat. Infect Immun. 1994;62(11):4755-4761.

39. Brunet K, Alanio A, Lortholary O, Rammaert B. Reactivation of dormant/latent fungal infection. J Infect. 2018;77(6):463-468.

40. Murakami K, et al. Knock out of S1P3 receptor signaling attenuates inflammation and fibrosis in bleomycin-induced lung injury mice model PLOS ONE. 2014;9(9):e106792.

41. Sanna MG, et al. Sphingosine 1-phosphate (S1P) receptor subtypes S1P1 and S1P3, respectively, regulate lymphocyte recirculation and heart rate. J Biol Chem. 2004;279(14):13839-13848.

42. Blaho VA, Hla T. An update on the biology of sphingosine 1-phosphate receptors. J Lipid Res. 2014;55(8):1596-1608.

43. Hou J, et al. S1PR3 Signaling drives bacterial killing and is required for survival in bacterial sepsis. Am J Respir Crit Care Med. 2017;196(12):1559-1570.

44. Müller J, von Bernstorff W, Heidecke CD, Schulze T. Differential S1P receptor profiles on M1- and M2-polarized macrophages affect macrophage cytokine production and migration. Biomed Res Int. 2017;2017:7584621.

45. Hardison SE, Ravi S, Wozniak KL, Young ML, Olszewski MA, Wormley FL. Pulmonary infection with an interferon-gamma-producing Cryptococcus neoformans strain results in classical macrophage activation and protection. Am J Pathol. 2010;176(2):774-785.

46. Anastasiadou S, Knöll B. The multiple sclerosis drug fingolimod (FTY720) stimulates neuronal gene expression, axonal growth and regeneration. Exp Neurol. 2016;279:243-260.

47. Zhu L, Jones C, Zhang G. The role of phospholipase $\mathrm{C}$ signaling in macrophage-mediated inflammatory response. JImmunol Res. 2018;2018:5201759.

48. Nussbaum C, et al. Sphingosine-1-phosphate receptor 3 promotes leukocyte rolling by mobilizing endothelial P-selectin. Nat Commun. 2015;6:6416.

49. Sugimoto N, Takuwa N, Okamoto H, Sakurada S, Takuwa Y. Inhibitory and stimulatory regulation of Rac and cell motility by the G12/13-Rho and Gi pathways integrated downstream of a single $G$ protein-coupled sphingosine-1-phosphate receptor isoform. Mol Cell Biol. 2003;23(5):1534-1545.

50. Gaire BP, Song MR, Choi JW. Sphingosine 1-phosphate receptor subtype $3\left(\mathrm{~S}_{3} \mathrm{P}_{3}\right)$ contributes to brain injury after transient focal cerebral ischemia via modulating microglial activation and their M1 polarization. J Neuroinflammation. 2018;15(1):284.

51. Shen $\mathrm{Y}$, et al. S1P/S1PR3 axis promotes aerobic glycolysis by YAP/c-MYC/PGAM1 axis in osteosarcoma. EBioMedicine. 2019;40:210-223.

52. Sun RZ, et al. Rapamycin and FTY720 alleviate atherosclerosis by cross talk of macrophage polarization and autophagy. Biomed Res Int. 2018;2018:1010248.

53. Qin C, et al. Fingolimod protects against ischemic white matter damage by modulating microglia toward M2 polarization via STAT3 pathway. Stroke. 2017;48(12):3336-3346.

54. Khan A, Singh VK, Hunter RL, Jagannath C. Macrophage heterogeneity and plasticity in tuberculosis. J Leukoc Biol. 2019;106(2):275-282. 55. Ward MD, Jones DE, Goldman MD. Cryptococcal 
meningitis after fingolimod discontinuation in a patient with multiple sclerosis. Mult Scler Relat Disord. 2016;9:47-49.

56. Seto H, et al. Disseminated Cryptococcosis in a 63-year-old patient with multiple sclerosis treated with fingolimod. Intern Med. 2016;55(22):3383-3386.

57. Lazzarini C, et al. Acylhydrazones as antifungal agents targeting the synthesis of fungal sphingolipids. Antimicrob Agents Chemother. 2018;62(5):e00156-18.

58. Kono M, et al. The sphingosine-1-phosphate receptors $\mathrm{S} 1 \mathrm{P} 1, \mathrm{~S} 1 \mathrm{P} 2$, and $\mathrm{S} 1 \mathrm{P} 3$ function coordi- nately during embryonic angiogenesis. J Biol Chem. 2004;279(28):29367-29373.

59. Anderson KG, et al. Intravascular staining for discrimination of vascular and tissue leukocytes. Nat Protoc. 2014;9(1):209-222.

60. Mori $\mathrm{H}$, et al. Introduction of Zinc-salt fixation for effective detection of immune cell-related markers by immunohistochemistry. Toxicol Pathol. 2015;43(6):883-889.

61. Chi V, Chandy KG. Immunohistochemistry: paraffin sections using the Vectastain $\mathrm{ABC}$ kit from vector labs. J Vis Exp. 2007;(8):308.

62. Lawson WE, et al. Characterization of fibro- blast-specific protein 1 in pulmonary fibrosis. Am J Respir Crit Care Med. 2005;171(8):899-907.

63. Sheehan DC, Hrapchak BB. Theory and Practice of Histotechnology. The CV Mosby Company; 1980.

64. Casadevall A, et al. Characterization of a murine monoclonal antibody to Cryptococcus neoformans polysaccharide that is a candidate for human therapeutic studies. Antimicrob Agents Chemother. 1998;42(6):1437-1446.

65. Luberto C, et al. Identification of App1 as a regulator of phagocytosis and virulence of Cryptococcus neoformans. J Clin Invest. 2003;112(7):1080-1094. 